\title{
Myeloid cell-derived PROS1 inhibits tumor metastasis by regulating inflammatory and immune responses via IL-10
}

\author{
Avi Maimon, ${ }^{1}$ Victor Levi-Yahid, ${ }^{1}$ Kerem Ben-Meir, ${ }^{2}$ Amit Halpern, ${ }^{1}$ Ziv Talmi, ${ }^{1}$ Shivam Priya, ${ }^{1}$ Gabriel Mizraji, ${ }^{1}$ Shani Mistriel-Zerbib, ${ }^{2}$ \\ Michael Berger, ${ }^{2}$ Michal Baniyash, ${ }^{2}$ Sonja Loges, ${ }^{3,4}$ and Tal Burstyn-Cohen ${ }^{1}$ \\ ${ }^{1}$ The Institute for Dental Sciences, Faculty of Dental Medicine and ${ }^{2}$ The Lautenberg Center for Immunology and Cancer Research, Israel-Canada Medical Research Institute, Faculty of Medicine, the Hebrew \\ University, Jerusalem, Israel. ${ }^{3}$ Division of Personalized Medical Oncology (A420), German Cancer Research Center (DKFZ), Heidelberg, Germany. ${ }^{4}$ Department of Personalized Oncology, University Hospital \\ Mannheim, Mannheim, Germany.
}

\begin{abstract}
Stimulation of TAM (TYRO3, AXL, and MERTK) receptor tyrosine kinases promotes tumor progression through numerous cellular mechanisms. TAM cognate ligands CAS6 and PROS1 (for TYRO3 and MERTK) are secreted by host immune cells, an interaction which may support tumor progression. Here, we revealed an unexpected antimetastatic role for myeloid-derived PROS1: suppressing metastatic potential in lung and breast tumor models. Pros1 deletion in myeloid cells led to increased lung metastasis, independent of primary tumor infiltration. PROS1-cKO bone marrow-derived macrophages (BMDMs) led to elevated TNF- $\alpha$, IL-6, Nos2, and IL-10 via modulation of the Socs3/NF-кB pathway. Conditioned medium from cKO BMDMs enhanced EMT, ERK, AKT, and STAT3 activation within tumor cells and promoted IL-10-dependent invasion and survival. Macrophages isolated from metastatic lungs modulated T cell proliferation and function, as well as expression of costimulatory molecules on DCs in a PROS1-dependent manner. Inhibition of MERTK kinase activity blocked PROS1-mediated suppression of TNF- $\alpha$ and IL-6 but not IL-10. Overall, using lung and breast cancer models, we identified the PROS1/MERTK axis within BMDMs as a potent regulator of adaptive immune responses with a potential to suppress metastatic seeding and revealed IL-10 regulation by PROS1 to deviate from that of TNF- $\alpha$ and IL-6.
\end{abstract}

\section{Introduction}

Macrophages play dominant roles in tumor microenvironment (TME) interactions affecting tumor growth and metastasis. By secreting various factors, macrophages can alter tumor cell behavior, often promoting cancer initiation and supporting metastatic progression (1-4). The bidirectional cross-talk between tumor cells and macrophages was shown to modulate tumor growth and metastasis through several mechanisms (5). Examples include macrophage polarization toward an M2-like immunosuppressive phenotype by tumor-secreted lactic acid (6) and the induction of the proinflammatory cytokines TNF- $\alpha$ and IL- 6 within macrophages by tumor-derived versican, leading to increased metastasis (7). Another macrophage-mediated tumor-promoting pathway includes the secretion of molecules that activate proto-oncogenes expressed by tumor cells. This was shown for growth arrest-specific 6 (Gas6), which is upregulated by tumor-infiltrating macrophages. GAS6 leads to increased tumor growth and metastasis by stimulating its cognate receptors TYRO3, AXL, and MERTK, which constitute the TAM family of receptor tyrosine kinases (8), also identified as proto-oncogenes (9). We therefore reasoned that Protein S (PROS1), a TAM agonist with high homology to GAS6 also expressed by immune cells, would likewise activate TAM oncogenic signaling

Conflict of interest: The authors have declared that no conflict of interest exists. Copyright: ( 2021, American Society for Clinical Investigation.

Submitted: November 12, 2018; Accepted: April 7, 2021; Published: May 17, 2021.

Reference information: / Clin Invest. 2021;131(10):e126089.

https://doi.org/10.1172/JCl126089. through tumor-immune cell interactions. Because TME interactions modulate tumor growth and metastasis, identifying the factors underlying these interactions is of great importance.

As a ligand for the proto-oncogenic TAMs, we recently reported the upregulation of PROS1 in oral squamous cell carcinoma (OSCC), where PROS1 supported OSCC aggressiveness though AXL activation. Inhibition of PROS1 repressed tumor cell phenotypes both in vitro and in vivo (10), indicating its tumor-promoting capacity. In addition to their oncogenic capabilities, TAMs are key regulators of immune response and inflammation (11-14). Activation of TAMs in immune cells by their agonists GAS6 and PROS1 alleviates the immune response and mitigates inflammation (14-16). TAM inhibition is proinflammatory, as seen in mice after genetic inactivation of TAMs (17-20) and ligands $(15,16)$. Using a zymosan-induced model of peritonitis, we recently identified PROS1 as a regulator of peritoneal macrophage polarization that also mediates their phagocytic clearance of apoptotic neutrophils - both essential elements contributing to the resolution of inflammation (16).

In this study, we investigated the role of PROS1 in the myeloid compartment of the TME. We found that myeloid-derived PROS1 inhibits tumor metastasis through tempering peripheral inflammation and immune modulation rather than by directly stimulating TAM-dependent oncogenic signaling within tumor cells. Conditional knockout (cKO) of Pros1 in myeloid cells resulted in their hyperinflammatory profile and increased infiltration into metastatic lungs, as well as elevated levels of the extracellular degrading enzyme MMP9. Direct effects on tumor cells led to epithelial-mesenchymal transition (EMT) and improved survival 
A

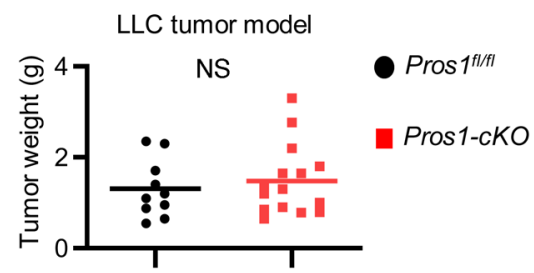

B

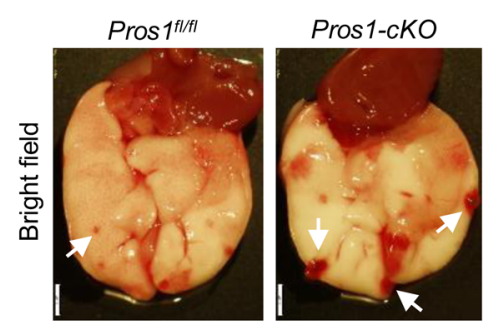

C
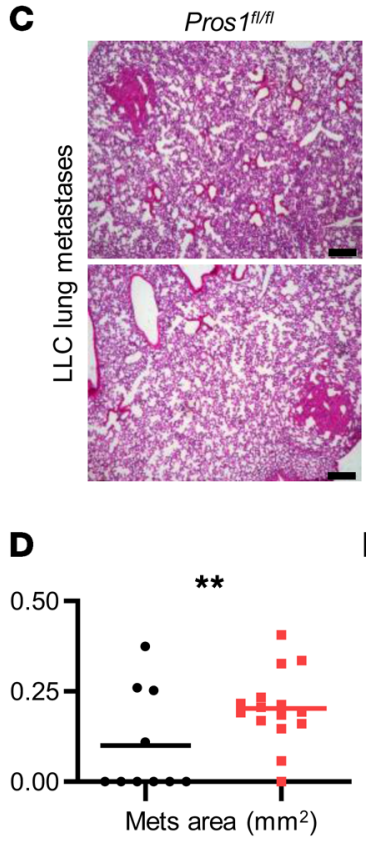

Pros1-cKO

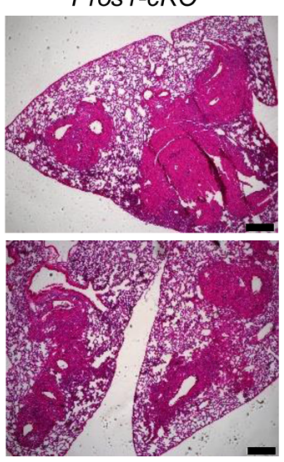

E

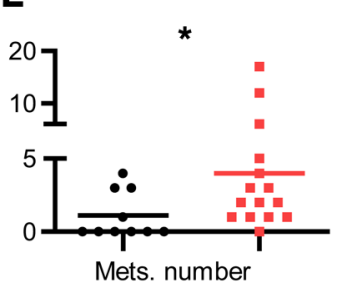

$\mathbf{F}$

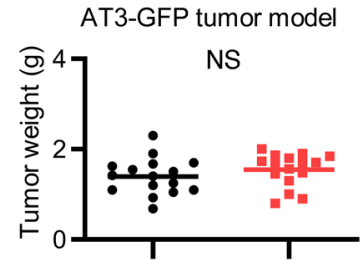

G
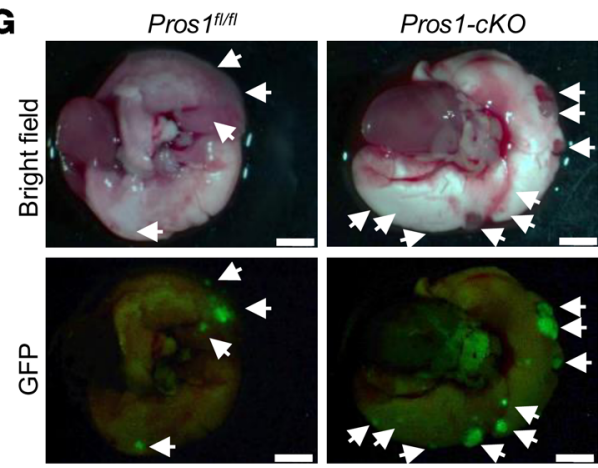

H

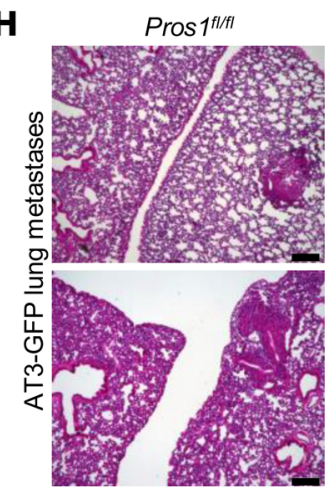

Pros1-cKO

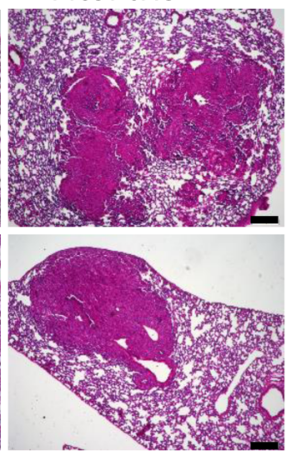

I

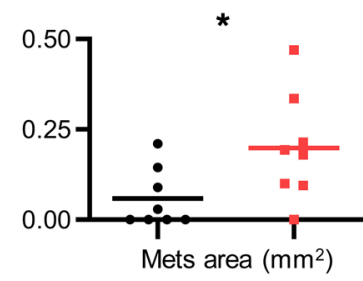

J

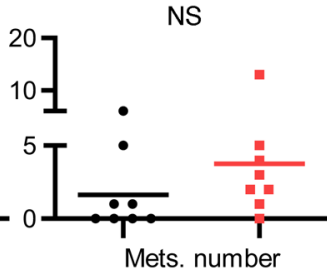

Pros $1^{f l \mid f i}$

Figure 1. Genetic ablation of Pros1 in host myeloid cells enhances metastasis. Analysis of Lewis lung carcinoma (LLC) (A-E) and AT3-GFP mammary (F-J) primary tumors and metastasis. ( $\mathbf{A}$ and $\mathbf{F}$ ) End-stage tumor weight $(n=10,15$ mice/group in $\mathbf{A}) ; n=16$ in $\mathbf{F}$. The horizontal line marks the mean tumor weight. (B and G) Lungs isolated 3 weeks after s.c. inoculation of $5 \times 10^{5}$ LLC cells (B) or after orthotropic injection of $1 \times 10^{6}$ AT3-CFP cells into each of 2 mammary glands (G). Metastatic foci appear as red nodules (arrows in B and $\mathbf{G}$ ). GFP-expressing AT3 metastatic foci appear green under fluorescent light (G, lower panels). Scale bars: $2 \mathrm{~mm}$. (C and $\mathbf{H})$ Representative images of H\&E-stained sections from lungs described in $\mathbf{B}$ and $\mathbf{G}$. Scale bars: $200 \mu \mathrm{m}$. Mean area ( $\mathbf{D}$ and $\mathbf{I}$ ) and number (E and $\mathbf{J})$ of metastases (Mets) scored. ${ }^{*} P<0.05$ (0.03 in $\mathbf{E}$ and 0.019 in $\left.\mathbf{I}\right),{ }^{* *} P=0.006$ (D); $t$ test. NS, nonsignificant.

and invasiveness. Concomitantly, inhibition of PROS1 expression affected DC maturation and $\mathrm{T}$ cell proliferation and activation. Together, our findings revealed PROS1 as a key player acting at multiple levels on both tumor cells and the TME, directly affecting metastasis. Moreover, our data indicate that PROS1 expression in myeloid cells dictates the inflammatory status of the lungs. We further show that PROS1 is a key molecular switch of inflammatory signaling within bone marrow-derived macrophages (BMDMs), modulating the NF- $\mathrm{B}$ pathway. Addition of PROS1 to Pros1-cKO BMDMs suppressed their proinflammatory signature and reverted their prometastatic capacity in vivo. Mechanistically, we revealed that inhibiting the MERTK kinase activity blocked PROS1-mediated suppression of TNF- $\alpha$ and IL-6, but did not affect that of IL-10. Investigating the relevance of the PROS1/ IL-10 axis revealed that IL-10 acted directly on tumor cells to increase their survival and invasive potential while inhibiting $\mathrm{T}$ cell proliferation at the same time, which was suppressed upon addition of PROS1 or by IL-10 neutralization. Taken together, our 

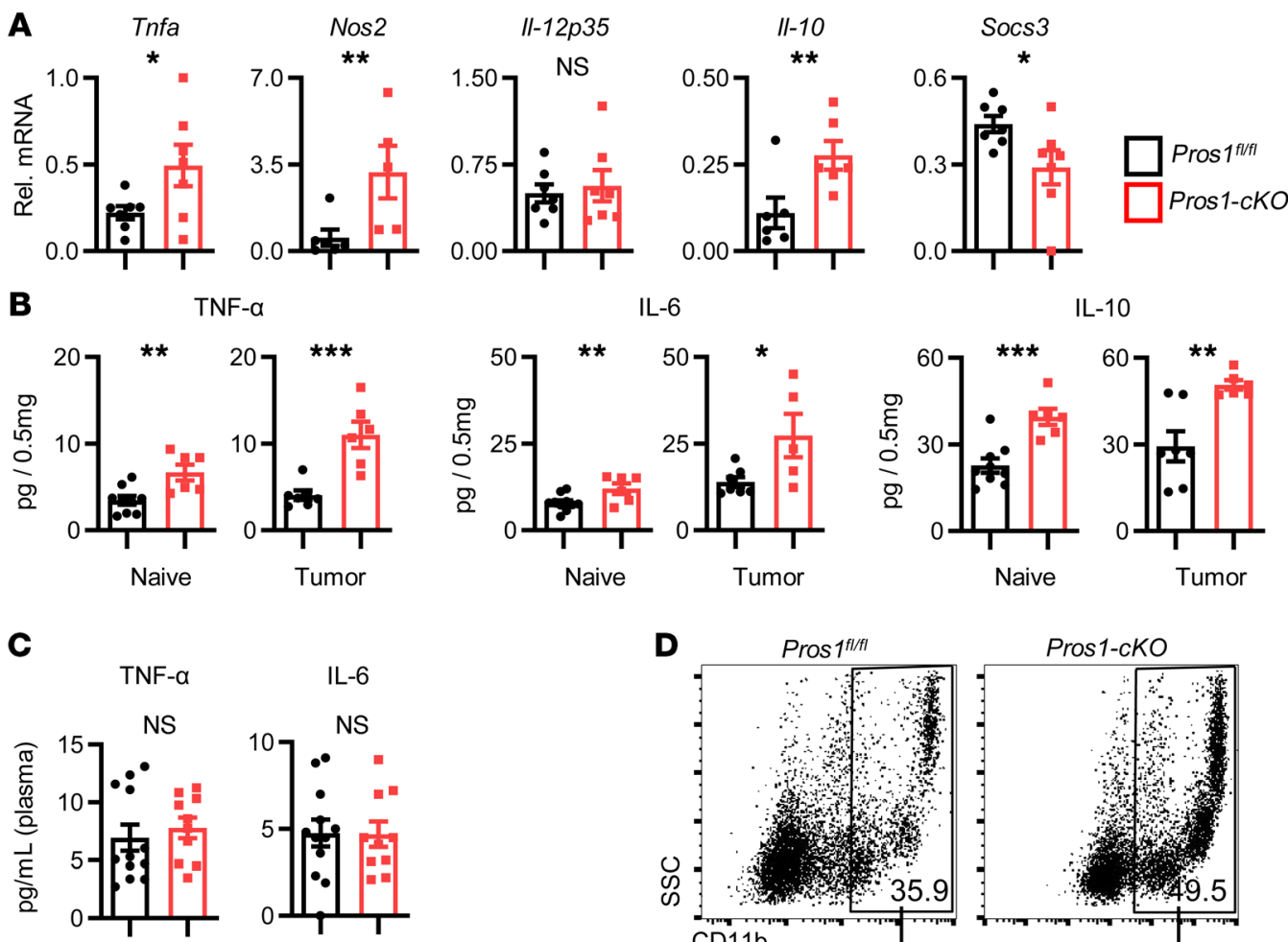

D
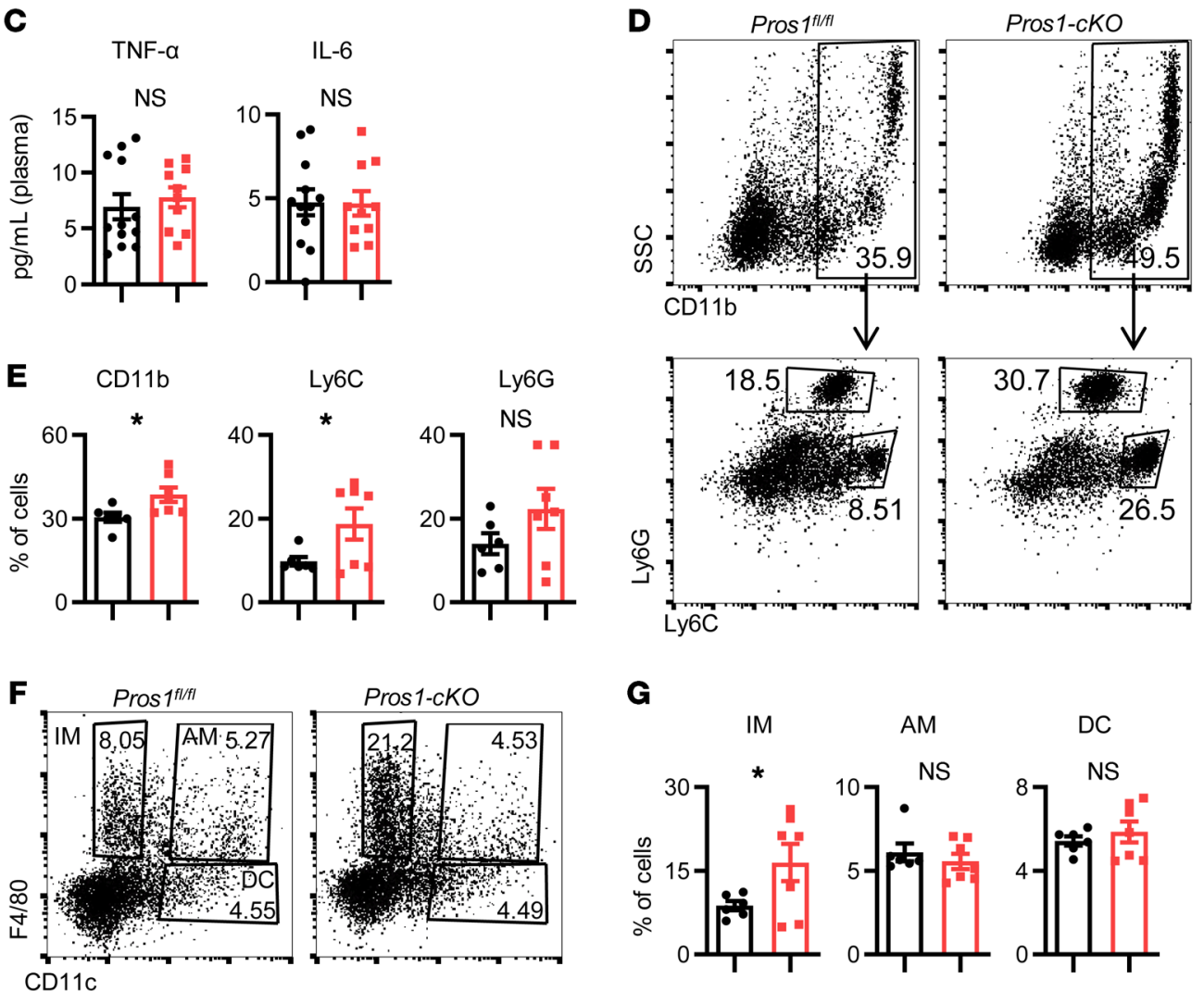

Figure 2. Characterization of lung inflammation in control and Pros1-cKO mice. (A and B) An elevated inflammatory signature is present in the lungs of mice after myeloid-specific ablation of Pros1. (A) Relative mRNA expression, measured by RT-qPCR, of the indicated cytokines from lungs of tumor-naive Pros $7^{f / f l}$ (black bars) and Pros1-cKO (red bars) mice. Mean \pm SEM, $n=5-7$ mice/group; 3 independent experiments. ${ }^{*} P \leq 0.02 ;{ }^{*} P \leq 0.01 ; t$ test. (B) Cytokines measured by ELISA from tumor-naive and tumor-bearing lung lysates. Mean $\pm \mathrm{SEM} ; n=5-10$ mice/group. ${ }^{*} P<0.05,{ }^{* *} P<0.01,{ }^{* * *} P<0.001(P$ $=0.002,0.009$, and 0.0003 and $P=0.0004,0.016,0.002$ for TNF- $\alpha$, IL-6, and IL-10 in naive and tumor-bearing lungs, respectively); $t$ test. (C) ELISA measurements of TNF- $\alpha$ and IL- 6 serum levels in control and cKO mice. Mean \pm SEM; $n=10-12$ mice/group. (D-C) FACS analysis of immune cell infiltration into the lungs of tumor-naive control and cKO mice. Representative plots (D) and quantification (E) of CD11 $\mathrm{b}^{+}$from CD45 gated cells (top), further analyzed for Ly6C+Ly6C ${ }^{10}$ (granulocytes) and Ly6C-Ly6C ${ }^{\text {hi }}$ (monocytes). $n=7$ mice/genotype. ${ }^{*} P<0.05\left(P=0.01\right.$ and 0.02 for CD11b ${ }^{+}$and Ly6C-Ly6Chi, respectively); $t$ test. Representative FACS plots (F) and quantification (G) of CD45+ gated cells stained for F4/80 and CD11c identifying 3 myeloid subpopulations: IM (F4/80+),

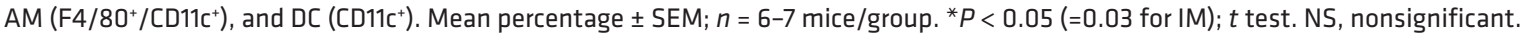

data identified PROS1 as a potentially novel antimetastatic protein acting at multiple levels. PROS1 (a) directly affects EMT and cancer cell survival, (b) modulates DC and T cell function, and (c) regulates inflammatory signaling in metastasis-associated macrophages, directly affecting the metastatic potential.

\section{Results}

Pros1 deficiency in host myeloid cells exacerbates tumor metastasis. To test whether PROS1 in host myeloid cells affects cancer progression, we genetically deleted Pros1 expression in the myeloid lineage by crossing $\operatorname{Pros}^{1 / / / l}$ mice (21) with $\mathrm{LysM}$-Cre $e^{+}$mice (22), 
efficiently driving Cre expression in myeloid cells. Pros ${ }^{1 / f l}$ (control) and $L y s M-\mathrm{Cre}^{+}$Pros $^{f l / f l}$-cKO mice (hereafter Pros1-cKO) were challenged with an s.c. injection of murine Lewis lung carcinoma (LLC) cells (23). Primary tumor growth dynamics in control and Pros1-cKO mice were comparable for the duration of the experiment (Figure 1A and Supplemental Figure 1A; supplemental material available online with this article; https://doi.org/10.1172/ JCI126089DS1). However, metastatic burden was significantly increased in the lungs of Pros1-cKO mice compared with their littermate controls (Figure 1, B-E). Histopathological analysis of lung sections revealed larger lung metastases in Pros1-cKO mice $\left(0.2 \pm 0.02 \mathrm{~mm}^{2}\right)$ compared with controls $\left(0.09 \pm 0.04 \mathrm{~mm}^{2}\right)$. Pros1-cKO lungs also showed increased numbers of metastatic foci $(4 \pm 1.2)$ compared with controls $(1.1 \pm 0.5$; Figure $1, \mathrm{C}-\mathrm{E})$. We also tested the effect of myeloid Pros1 expression on tumor growth and metastasis using the AT3 mammary carcinoma model (Figure 1, F-J). Akin to the lung cancer model, primary tumor growth of orthotopically injected GFP-expressing AT3 cells was similar between control and Pros1-cKO mice (Figure 1F and Supplemental Figure 1, B and C). Although there were no significant differences in the number of metastatic foci (Figure 1J), metastases in Pros1-cKO mice were larger $\left(0.198 \pm 0.05 \mathrm{~mm}^{2}\right)$ than in controls $\left(0.06 \pm 0.03 \mathrm{~mm}^{2} ;\right.$ Figure 1I). These results indicate that ablation of Pros 1 in host myeloid cells minimally affected primary tumor growth, but significantly enhanced metastasis of syngeneic models of mammary and lung cancers, indicating an inverse association between metastasis and host myeloid PROS1 expression. This association revealed an unanticipated, yet decisive antimetastatic role for host immune cell-derived PROS1.

Pros1 ablation in myeloid cells induces lung inflammation, increasing their permissiveness for tumor cell colonization. As a ligand for the TAMs, PROS1 takes a cell-autonomous negative regulatory function in the immune response of DCs and in the regulation of DCs by T cells (12-15). Inactivation of TAM signaling in mice results in a chronically activated immune system, autoimmune disease, and a proinflammatory cytokine signature $(12,20)$. These phenotypes notwithstanding, the inflammatory status of mice lacking PROS1 in the myeloid lineage and its possible impact on cancer have not been investigated. We hypothesized that lack of PROS1 in the myeloid compartment may cause systemic or local inflammation in the lungs, previously demonstrated to promote metastasis in several cancer models (24-26). Indeed, lungs of Pros1-cKO mice expressed higher baseline levels of Tnfa and Nos2, without changes in the p35 subunit of $I l-12$, along with decreased expression of Socs3 (Figure 2A). Interestingly, mRNA levels of the antiinflammatory cytokine $\mathrm{Il}-10$ were concomitantly elevated (Figure 2A). TNF- $\alpha$, IL-6, and IL-10 protein levels were also higher in cKO lungs, which became more pronounced after tumor challenge (Figure $2 \mathrm{~B}$ ). This proinflammatory signature was restricted to the lungs; TNF- $\alpha$ and IL- 6 protein in the plasma remained at basal levels and were similar between control and Pros1-cKO mice (Figure 2C).

Analysis of immune cell infiltration revealed elevated ratios of total myeloid cells $\left(\mathrm{CD} 11 \mathrm{~b}^{+}\right)$and monocytes (Ly6C $\left.{ }^{\text {hi }} \mathrm{Ly}_{6 \mathrm{G}}\right)$ in Pros1-cKO lungs, with a nonsignificant increase in granulocytes $\left(\right.$ Ly6 $\mathrm{G}^{+} \mathrm{Ly}_{6} \mathrm{C}^{\mathrm{lo}}$ ) (Figure 2, D and E). Pulmonary macrophages are heterogeneous; alveolar macrophages (AMs) residing in the airway lumen highly express the integrin CD11c but not CD11b, and interstitial macrophages (IMs) are located within the lung interstitial space together with DCs, another myeloid population expressing PROS1. IMs are distinguished from AMs by their high CD11b and F4/80 and low CD11c expression $(27,28)$. Whereas AM and DC populations were comparable between control and cKO mice, IM frequencies were significantly elevated in the lungs of $\mathrm{cKO}$ mice (Figure 2, F and G). Originating from circulating monocytes (29), IMs regulate lung immune homeostasis and provide immune-suppressive functions through elevated secretion of IL-10 (28). In contrast with the metastatic site, no differences were observed in monocyte $\left(\mathrm{Ly}_{6 \mathrm{C}} \mathrm{Ly}^{\mathrm{L}} \mathrm{G}\right)$, granulocyte $\left(\mathrm{Ly}_{6 \mathrm{G}} \mathrm{Ly}^{+} \mathrm{C}^{+}\right)$, or macrophage $\left(\mathrm{F} 4 / 80^{+}\right)$frequencies in the primary tumor (Supplemental Figure 2, A and B). Taken together, these results indicated that loss of PROS1 in the myeloid compartment was sufficient to induce lung inflammation.

We next tested whether inflammation in Pros1-cKO lungs provides a permissive environment for metastatic cells by assessing in vivo survival and colonization after i.v. injection of LLC-GFP ${ }^{+}$ cells. Dissemination and colony formation in Pros1-cKO lungs was enhanced 21 days later, with more superficial GFP-expressing metastatic foci (Supplemental Figure 2C). Scoring LLC-GFP ${ }^{+}$cells by FACS revealed that Pros1-cKO lungs had 3.6-fold more $\mathrm{GFP}^{+}$ cells compared with controls (Supplemental Figure 2D). Lungs from these Pros1-cKO mice also expressed higher Tnfa, Nos2, and Il-12p35 levels (Supplemental Figure 2E), correlative with increased colonization of the lungs. We found that inflammation in Pros1-cKO lungs preceded metastatic seeding; elevated Tnfa, Il6, and Nos 2 production was recorded as early as 10 days after inoculation, prior to any visually detected metastases, and remained elevated with the addition of $I l-10$ at 20 days after inoculation (Supplemental Figure 2, F and G). Thus, our results indicate that inflamed lungs of Pros1-cKO mice support enhanced colonization and metastasis formation by LLC cells. Similar results after i.v. injection imply metastasis is independent of any interactions at the primary tumor site.

Expression of Pros1 in lung-infiltrating bone marrow-derived myeloid cells determines lung inflammation. We hypothesized that the elevated frequencies of Pros1-deficient myeloid cells provoking a protumor effect originate from the circulation, as seen in a variety of cancer models $(1,3,11,30)$. Particularly in LLCs, infiltrating macrophages exacerbate lung metastasis (31), whereas macrophage deficiency attenuates LLC tumor growth and metastasis in osteopetrotic (op/op) mice lacking CSF-1 (32). Monocytes and tumor-associated macrophages also facilitate metastasis in a PyMT mammary tumor model (33-35). Bone marrow-derived (BM-derived) inflammatory monocytes, IMs, and AMs all support a prometastatic niche $(33,36,37)$. To understand whether metastatic lungs display the same immune-infiltrating profile as tumor-naive mice, $\mathrm{F} 4 / 80^{+}$cells were sorted from tumor-harboring mice and characterized immunologically and for their cytokine expression. Similar to naive cKO lungs that had elevated IMs (Figure 2, $\mathrm{F}$ and $\mathrm{G}$ ), $\mathrm{F} 4 / 80^{+}$cells from $\mathrm{cKO}$ metastatic lungs expressed CD11b ${ }^{\text {hi }}$ but very low CD11c, resembling the IM profile (Figure 3, A and $\mathrm{B}$ ). Within $\mathrm{F} 4 / 80^{+}$sorted cells from metastatic $\mathrm{CKO}$ mice, the fraction of Ly6 $\mathrm{C}^{\text {hi }}$ increased, along with a decrease in Ly $6 \mathrm{C}^{\text {lo }}$ cells (Figure 3B), indicating a trend toward monocyte (Ly6C $\left.\mathrm{C}^{\text {hi }}\right)$ enrich- 
A
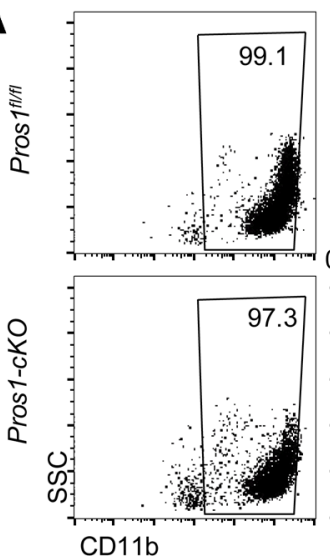
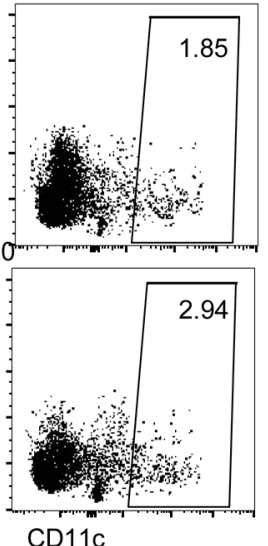

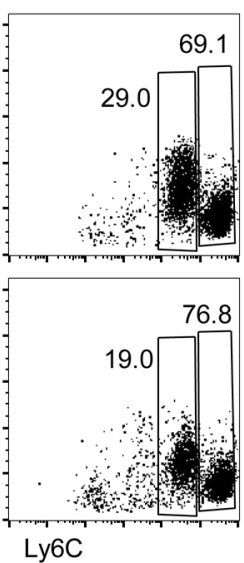

B

$\mathrm{CD} 11 \mathrm{~b}^{+} / \mathrm{CD} 11 \mathrm{C}^{+} \quad$ Ly6C $\mathrm{C}^{\mathrm{i} / L y 6 \mathrm{C}^{\circ}}$
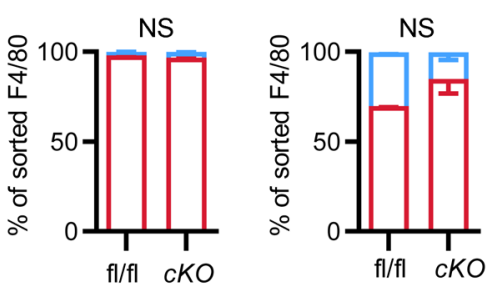
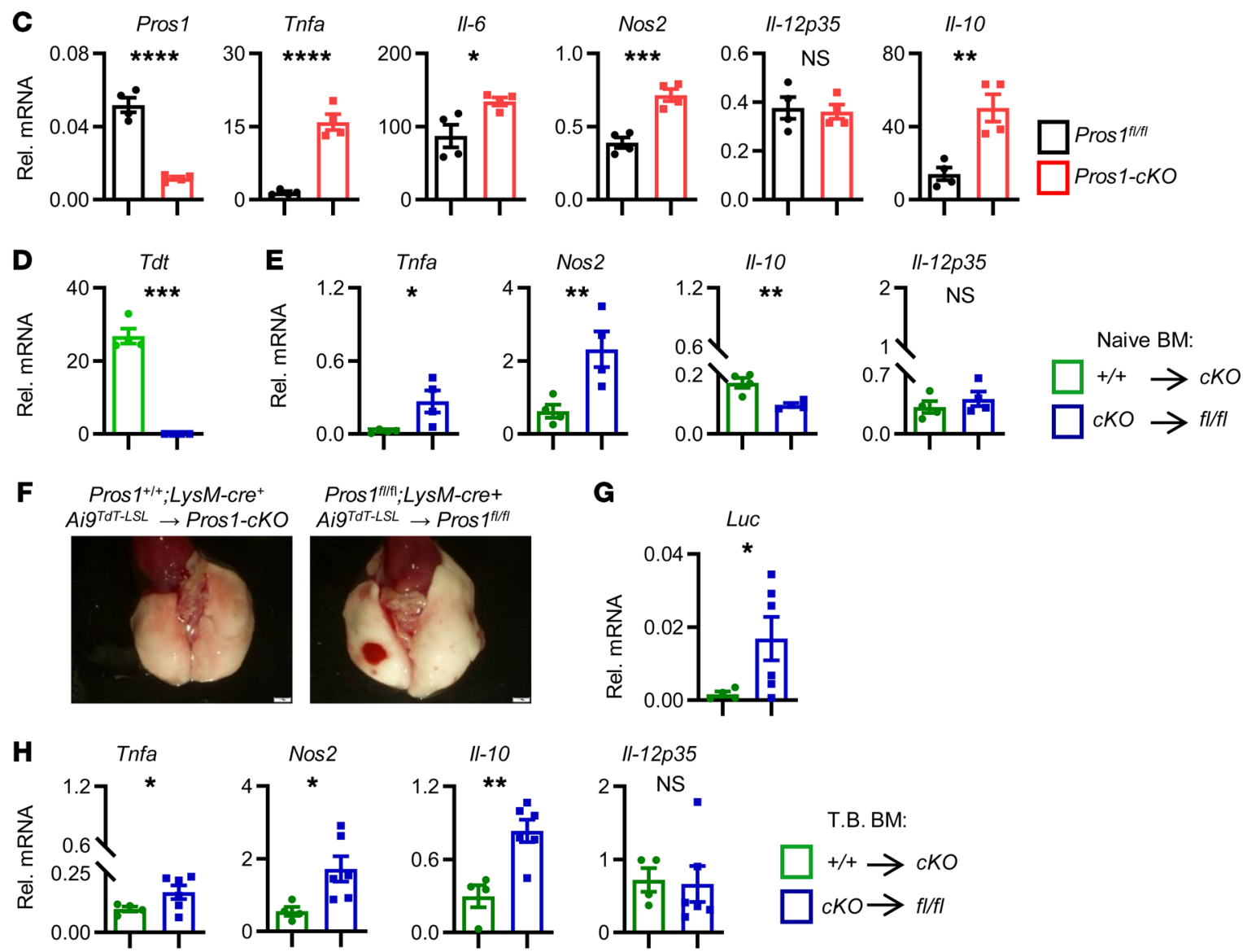

Figure 3. Pros1-deficient F4/80+ bone marrow-derived cells infiltrate metastatic lungs and impose inflammation in vivo. (A-C) Analysis of lung-infiltrating immune cells in tumor-bearing mice. F4/80+ cells sorted from metastatic lungs were characterized immunologically (A and B) and by mRNA expression (C). (B)

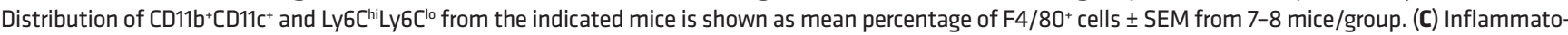
ry cytokine analysis by RT-qPCR of F4/80+ cells isolated from metastatic lungs. Each symbol represents a pool of 7-8 mice/group; 3 independent experiments. Mean relative values \pm SEM. ${ }^{*} P<0.05,{ }^{* *} P<0.01$, ${ }^{* * *} P<0.001$, ${ }^{* * *} P<0.0001(P=0.014,0.0005$, and 0.002 for $I I-6$, Nos2, and $I I-10$, respectively); $t$ test. (D-H) Adoptive transfer of Pros1-deficient bone marrow (BM) induces lung inflammation and supports lung metastasis. (D and E) TdT and cytokine expression in lungs 7 weeks after BM transplantation from Pros $7^{+/+} \mathrm{TdT}^{+}$into TdT-deficient cKO (green bars) and TdT ${ }^{+}-\mathrm{cKO}$ into TdT-deficient Pros $7^{\mathrm{fl} / \mathrm{fl}}$ (fl/fl, blue bars) in a tumor-naive setting. Mean \pm SEM; 4 mice/group. ${ }^{*} P<0.05$, ${ }^{* *} P<0.01$, ${ }^{* * *} P<0.001$, ${ }^{* * *} P<0.0001(P=0.018,0.008$, and 0.003 for Tnfa, Nos2, and II-10, respectively); $t$ test. $(\mathbf{F}-\mathbf{H})$ LLC-Luc cells were s.c. injected 4 weeks after BM transplantation. Three weeks later, lungs were isolated (F) and analyzed by RT-qPCR $(\mathbf{G}$ and $\mathbf{H})$. Mean \pm SEM; $n=4-6$ mice/group. ${ }^{*} P \leq 0.05,{ }^{* *} P<0.01(P=0.002$ for $I I-10) ; t$ test. NS, nonsignificant.

ment with a higher proinflammatory profile (38). The expression of Tnfa, Il-6, Nos2, and Il-1O in F4/80+ macrophages sorted from cKO but not control lungs confirmed their inflammatory nature in an in vivo metastatic setting (Figure $3 \mathrm{C}$ ).
We next sought to test whether BM-derived cells can modify lung inflammation in a Pros1-dependent manner. For this, the $\mathrm{BM}$ of lethally irradiated control and Pros1-cKO mice was reconstituted with BM from the reciprocal genotype. Pros1-express- 


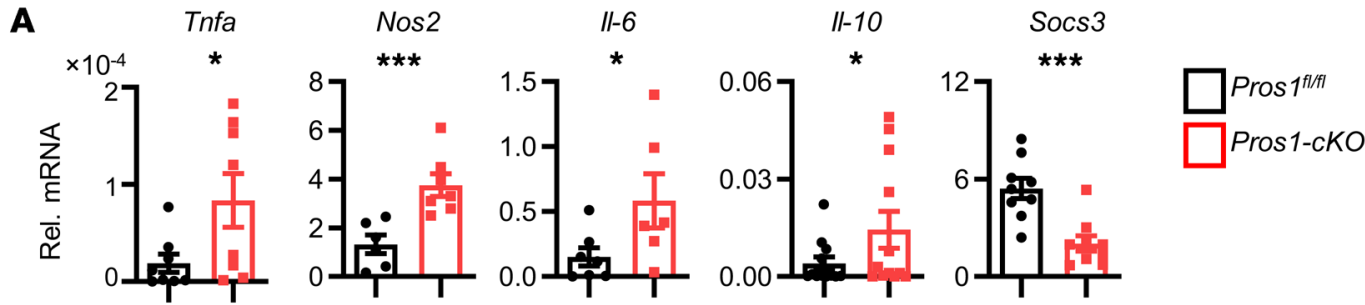

B

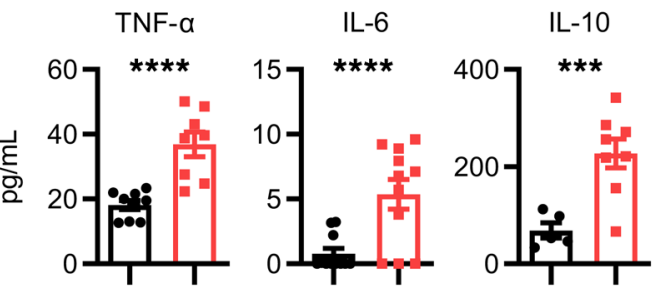

C

Mertk
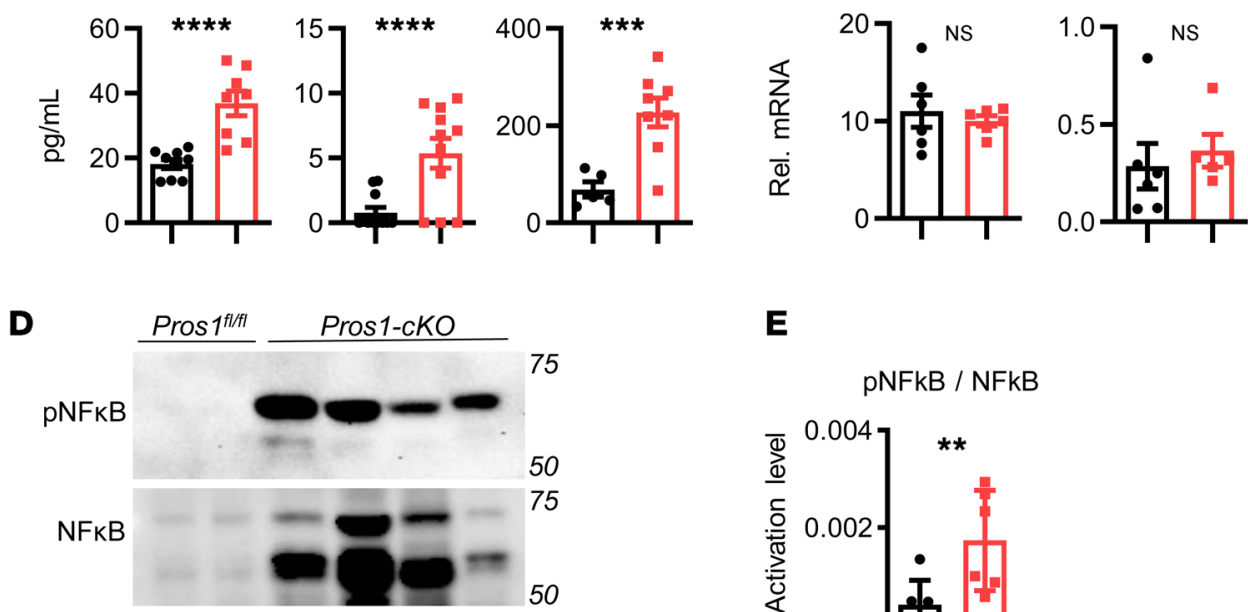

$\mathbf{E}$

pNFkB / NFkB

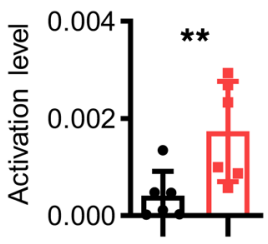

GAPDH

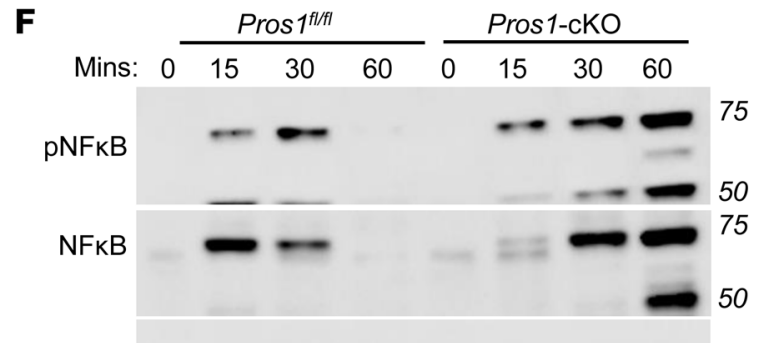

GAPDH
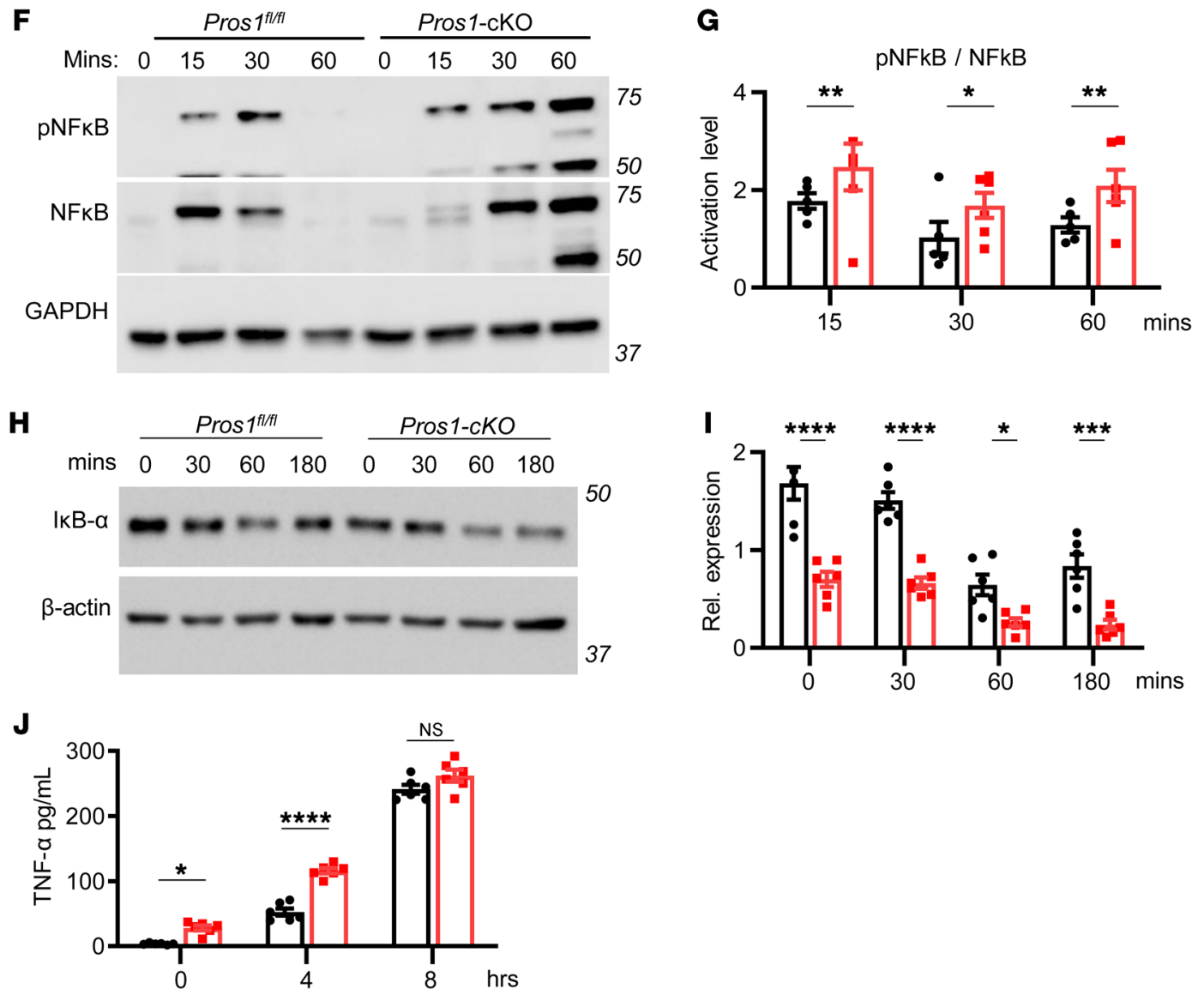
Figure 4. Pros1-deficient macrophages exhibit a proinflammatory profile regulated by the NF-KB pathway. (A) Cytokine transcript levels from cultured BMDMs derived from tumor-naive control and Pros1-cKO mice measured by RT-qPCR. Relative mean expression \pm SEM, $n=6-12$ mice/group. ${ }^{*} P<0.05$, ${ }^{* * *} P \leq 0.001$ ( $P=0.02,0.001,0.03,0.049$, and 0.0003 for Tnfa, Nos2, II-6, II-10, and Socs3, respectively); $t$ test. (B) ELISA measurements of TNF- $\alpha$, IL-6, and IL-10 from BMDM-CM isolated from tumor-naive Pros1-cKO and control mice. Average cytokine values \pm SEM are shown for 8-11 mice. ${ }^{* *} P \leq 0.001$, ${ }^{* * *} P \leq$ 0.0001 ( $P=0.0001,0.0006$, and 0.001 for TNF- $\alpha$, IL-6, and IL-10, respectively); $t$ test. (C) Gas6 and MerTK expression in control and Pros1-ablated BMDMs; $n$ = 5-6 mice/group; $t$ test. (D and E) Representative Western blot analysis of pNF- $\kappa B$ and NF- $\kappa B$ from BMDMs at steady state (grown in $10 \% \mathrm{FBS}$ ), and (E) band intensities ( $n=6 /$ group). ${ }^{* *} P<0.01(=0.009)$. (F-I) Western blot analysis of the NF- $\kappa B$ pathway. (F) Representative blots of cell lysates showing pNF$\kappa \mathrm{B}, \mathrm{NF}-\kappa \mathrm{B}$, and band intensities (C) $\left({ }^{*} P=0.04,{ }^{*} P=0.01\right)$ of LPS-stimulated $(100 \mathrm{ng} / \mathrm{mL}$ ) serum-starved BMDMs derived from tumor-naive control and Pros1-cKO mice and of $\mathrm{I} \kappa \mathrm{B} \alpha\left(\mathbf{H}\right.$ and $\left.\mathbf{I} ;{ }^{*} P=0.05,{ }^{* *} P=0.001,{ }^{* * *} P<0.0001\right)$. $\beta$-Actin served as loading control. Graphs indicate the mean $\pm \mathrm{SEM} ; n=6$; 2-way ANOVA. (J) ELISA measurements of TNF- $\alpha$ production by control and Pros1-cKO serum-starved and LPS-treated $(500 \mathrm{ng} / \mathrm{mL}) \mathrm{BMDMs}$ at 0,4 , and 8 hours after LPS challenge. Average $\pm \mathrm{SEM} ; n=6-8$ mice. ${ }^{*} P=0.048$, ${ }^{* * *} P<$ 0.0001; 2-way ANOVA. NS, nonsignificant.

ing BM control (LysM-Cre ${ }^{+}$Pros $^{1^{+/+}}$Ai9 $\left.{ }^{\text {TdT-LSL }}\right)$ carried the tomato red reporter (Ai9 $9^{\text {TdT-LSL }}$ ) for monitoring reconstitution efficiency. Under such conditions, the recipient BM population is lethally irradiated, whereas the radioresistant AMs are relatively unaffected (39). Eight weeks later, we verified efficient reconstitution by $\mathrm{Td} \mathrm{T}$ expression in the circulation of recipient mice. Analyzing the blood of recipient cKO mice reconstituted with TdT-labeled PROS1-expressing BM (Prosi $\left.{ }^{+/+} \mathrm{LysM}-\mathrm{Cre}^{+}{ }^{\mathrm{TdT}} \rightarrow \mathrm{CKO}\right)$ revealed $\mathrm{TdT}$ signal resembling the endogenous TdT levels in naive mice (Supplemental Figure 3, A-D). Moreover, the lungs of transplanted cKO mice were infiltrated by TdT-expressing donor myeloid cells (Figure 3D). Assessing the inflammatory status of $\mathrm{cKO} \rightarrow-$ $\operatorname{Pros}^{1 / f / l}$ lungs revealed elevated Tnfa and Nos2 transcripts with low Il-1O levels (Figure 3E, blue bars). By contrast, lungs of cKO mice reconstituted with TdT-labeled Prosl ${ }^{+/+} \mathrm{BM}\left(\mathrm{Pros}^{1^{+/}} \mathrm{LysM}_{-}\right.$ $\mathrm{Cre}^{+} \mathrm{TdT}^{\mathrm{T}} \rightarrow \mathrm{CKO}$ ) had low levels of proinflammatory cytokines and higher Il-1O levels (Figure 3E, green bars), even though they had expressed high Tnfa and Nos2 levels prior to BM transfer (Figure $2 \mathrm{~A}$ ). The spleens of transplanted mice were also successfully populated by donor cells with similar induction of Tnfa, although Nos2 levels remained unaffected (Supplemental Figure 3E), indicating a differential effect for BM-derived PROS1 on spleen and lung tissue. Thus, PROS1-expressing myeloid cells transplanted into inflamed cKO hosts (Figure 3, D and E) reversed their proinflammatory profile, and Pros1-deficient BM was reciprocally sufficient to elevate lung inflammation in otherwise noninflamed control mice. To determine whether the inflammatory status of the lungs directly contributes to the metastatic burden, we challenged reciprocally transplanted mice with LLC-Luc ${ }^{+}$cells and assessed metastasis. Successful reconstitution in tumor-bearing mice was verified by TdT expression (Supplemental Figure 3F). At endpoint, cKO mice transplanted with control BM had less metastasis, whereas the reciprocally transplanted mice (controls receiving $\mathrm{CKO} \mathrm{BM}$ ) had elevated occurrences of metastasis, seen superficially and evidenced by luciferin expression (Figure 3, F and $\mathrm{G}$ ). As for tumor-naive transplanted mice, cytokine expres- sion in tumor-bearing control mice transplanted with cKO BM was elevated. However, compared with tumor-naive mice, the magnitude of inflammation was elevated, pointing to the contribution of metastatic cells to the overall inflammatory profile (Figure $3 \mathrm{H}$ ). Specifically, $I l-1 O$ levels significantly increased in tumor-bearing control mice that received $\mathrm{CKO} \mathrm{BM}$, suggesting a positive role for LLC cells in IL-10 expression within the TME.

Taken together, our results show that IMs are a myeloid-specific BM-derived population affecting lung inflammation in a PROS1-dependent manner, and show that the inflammatory status of the lungs was a major factor contributing to lung metastasis.

PROS1 reduces inflammatory cytokine production in BMDMs through regulation of the NF- $\kappa B$ pathway. The observation that PROS1-deficient BM-derived $\mathrm{F} 4 / 80^{+}$infiltrating cells are proinflammatory prompted us to investigate the mechanism by which PROS1 regulates inflammation in these cells. For this, we generated BMDMs from control (BMDM-fl/fl) and Pros1-cKO (BMDMcKO) mice (Supplemental Figure 4). BMDM-cKO mice exhibited higher transcript levels of Tnfa, Nos2, Il-6, and Il-10 and lower Socs3 mRNA (Figure 4A). TNF- $\alpha$, IL-6, and IL-10 cytokine secretion was also higher in BMDM-cKO mice (Figure 4B). Given the possible compensatory and redundant functions of GAS6 and MERTK in macrophages (20, 40-42), we measured expression of both genes in BMDM-cKO mice, which were unchanged (Figure 4C).

To dissect the molecular pathway underlying PROS1-mediated regulation of inflammation, we monitored the activation status of $\mathrm{NF}-\mathrm{\kappa B}$, a key regulator of cytokine production and previously shown to function downstream of TAM signaling (41), including in monocytes (17). Inhibition of NF-KB activation by GAS6 was shown in BM-derived DCs (BMDCs) (14) and in spleen-derived DCs by PROS1 secreted from T cells (15). However, the effect of PROS1 on NF-KB in macrophages is unknown. Under baseline conditions, pNF-kB levels were significantly elevated in BMDM-cKO macrophages (Figure 4, D and E), consistent with elevated TNF- $\alpha$, IL-6, and decreased Socs 3 levels recorded in these cells (Figure 4, A and B).

To assess the dynamics of NF- $\mathrm{kB}$ phosphorylation, serumstarved BMDMs were stimulated with LPS. In contrast with control cells, where NF- $\kappa \mathrm{B}$ activation was transient, in BMDM-cKO cells, high levels of pNF- $\mathrm{kB}$ persisted 1 hour after stimulation (Figure 4, F and G). Additionally, levels of the NF- $\kappa B$ inhibitor I $\kappa B \alpha$ were significantly lower in BMDM-cKO cells (Figure 4, $\mathrm{H}$ and I). Notably, the basal levels of I $\kappa \mathrm{B} \alpha$ at steady state (time point 0 ) were significantly lower in BMDM-cKO cells (Figure $4, \mathrm{H}$ and I). In line with hyperactivation of NF- $\mathrm{kB}$ in BMDM-cKO cells, the secretion of TNF- $\alpha$ was more rapidly induced upon LPS stimulation in cKO cells. This effect was toned down with time such that no differences in TNF- $\alpha$ secretion were observed 8 hours after LPS stimulation (Figure 4J). These results agree with the overall inflammatory profile observed in the lungs of Pros1-cKO mice (Figure 2 and Figure 3C), demonstrating for the first time, to our knowledge, that PROS1 is a negative regulator of the NF- $\mathrm{KB}$ pathway in macrophages and that the loss of PROS1 in BMDMs induces a proinflammatory transcriptional program.

PROS1 deletion in macrophages exacerbates lung metastasis through secreted factors. Inflammation plays a major role in tumor progression $(25,43,44)$, and loss of PROS1 in macrophages renders BMDMs and metastasis-associated macrophages hyperin- 
A

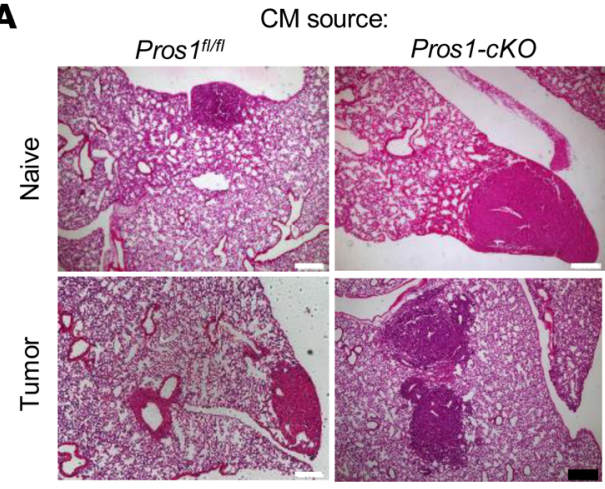

D

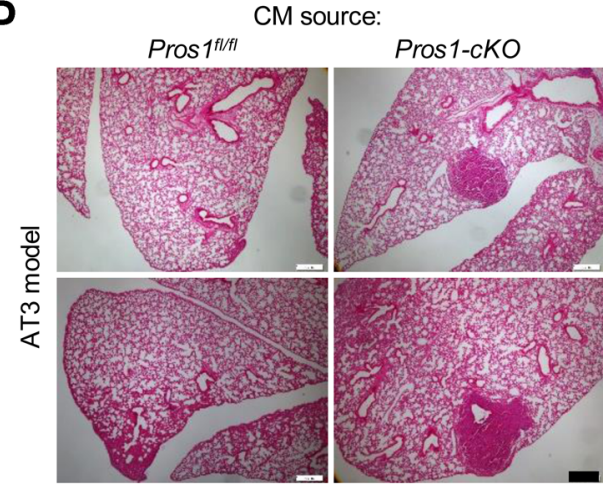

G

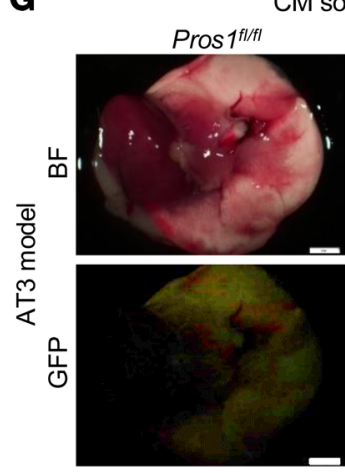

B

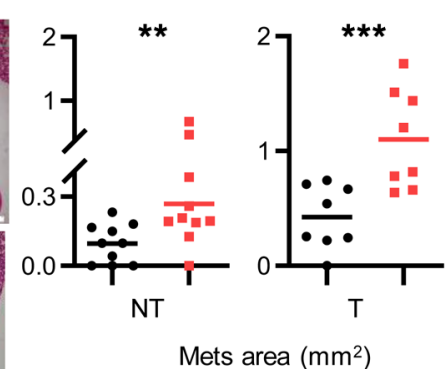

E

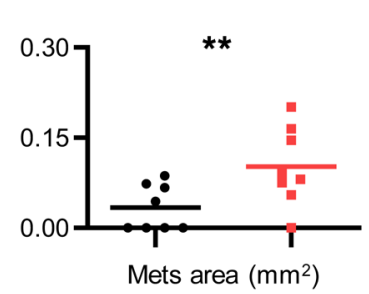

C
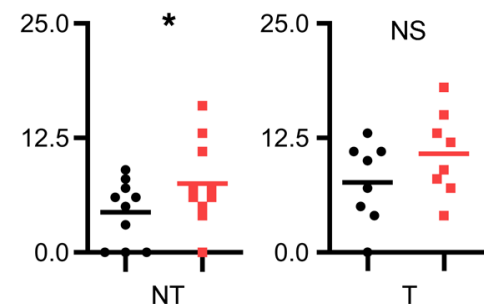

Mets number

$\mathbf{F}$

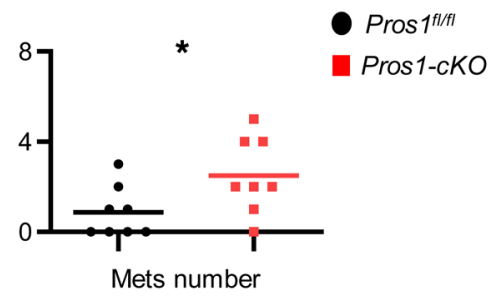

H

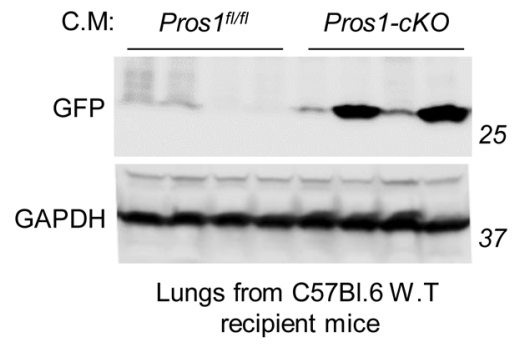

Figure 5. Enhanced metastatic potency of Pros1-deficient BMDMs. (A-C) LLC cells were educated for 24 hours with conditioned medium (CM) of BMDMs isolated from nontumor (NT) or tumor-bearing (T) control (Pros $f^{f / f l}$ ) and Pros1-cKO mice. Educated cells were injected into WT mice. Lung metastases (Mets) were evaluated at 3 weeks by H\&E. Representative H\&E-stained lung sections $(\mathbf{A})$, average area $\left(\mathbf{B} ;{ }^{* *} P=0.01\right.$ for NT; ${ }^{* * *} P=0.001$ for T), and number of metastases (C; ${ }^{*} P=0.05$ for NT; nonsignificant for T) are plotted. $n=8-10$ mice/group; $t$ test. (D-F) GFP-labeled AT3 cells were educated for 96 hours with the indicated BMDM-CM and injected into host WT mice. Lung metastases were evaluated 3 weeks later. $n=8$ mice/group. Representative H\&E lung sections (D), average metastatic area (E), and number (F) are shown. The horizontal line represents the mean value. ${ }^{*} P<0.05(=0.02)$, ${ }^{*} P=0.01 ; t$ test. Scale bars: $200 \mu \mathrm{m}$. (G and $\left.\mathbf{H}\right)$ Representative images of freshly isolated lungs described in $\mathbf{D}$ as observed under bright field (BF) and fluorescent illumination (GFP). Arrows indicate metastatic foci. Scale bars: 2 mm. (H) Representative Western blot for GFP content in lungs shown in G. GAPDH was used as a loading control. $n=4 /$ group.

flamed (Figure 3C and Figure 4), suggesting that loss of PROS1 in macrophages alone may exacerbate cancer progression through cytokine secretion. We therefore tested the ability of conditioned medium (CM) collected from tumor-naive BMDM-cKO cultures to affect LLC tumor progression and metastasis. LLC cells that were educated for 24 hours were s.c. injected into WT host mice expressing normal levels of PROS1 in all cells to exclude host-dependent effects and allow any changes in LLC tumor characteristics to be attributed to their education during the incubation with CM. In this experimental setup, no effect was observed on primary tumor growth, regardless of whether CM from control or BMDM-
cKO cultures was used for education (Supplemental Figure 5, A and B). By contrast, compared with cells educated by CM-BMDM-fl/fl, LLCs educated by CM-BMDM-cKO generated larger $(0.27 \pm 0.06$ $\mathrm{mm}^{2}$ versus $0.09 \pm 0.02 \mathrm{~mm}^{2}$ ) and more numerous ( $7.5 \pm 1.4$ versus $4.4 \pm 1$ ) metastases (Figure $5, \mathrm{~A}-\mathrm{C}$ ). Thus, our results indicate that BMDM-cKO elicited prometastatic features on tumor cells through secreted factors defined by a proinflammatory profile.

To determine whether the prometastatic effect of CM-BMDMcKO is influenced by the tumor status of the mice, we performed a similar experiment by using BMDMs from tumor-bearing mice. As with tumor-naive CM-BMDMs, no differences were observed 
A

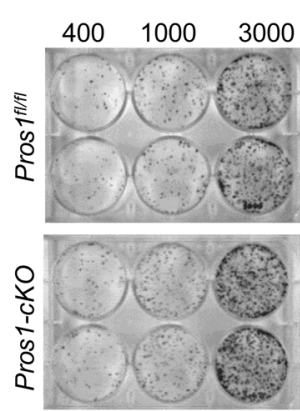

B

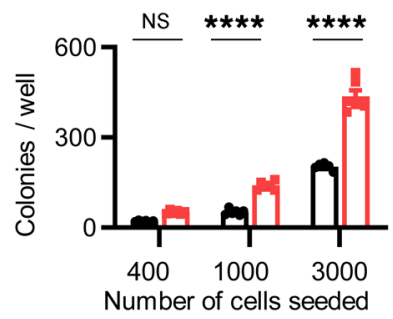

C

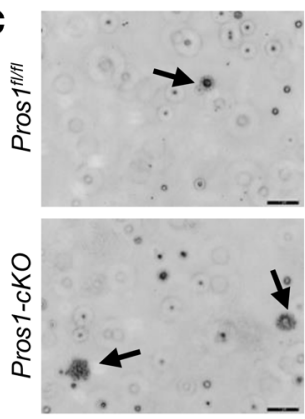

D

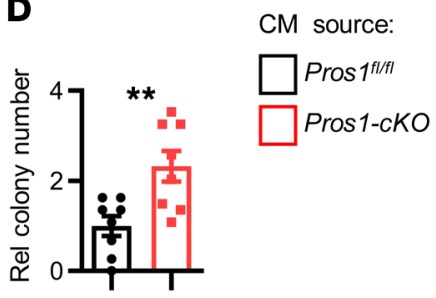

E

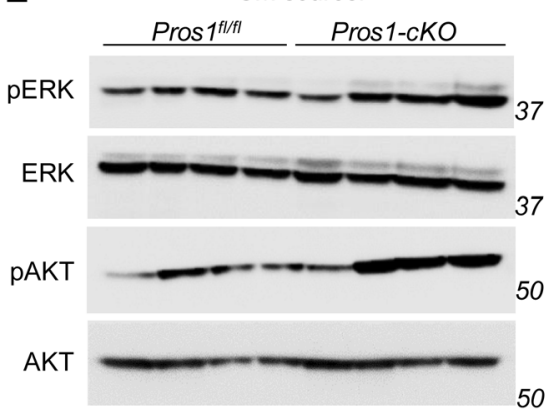

F pERK/ERK PAKT/AKT
G

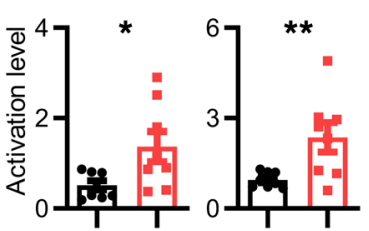

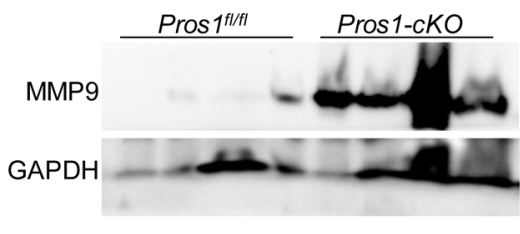

H

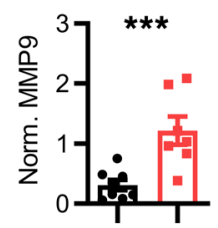

I

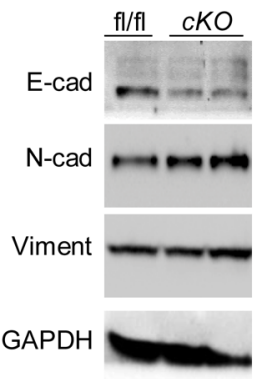

J E-Cadherin
$\mathrm{N}-$ Cadherin
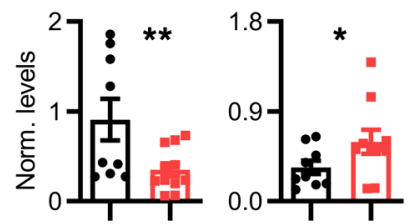

Vimentin

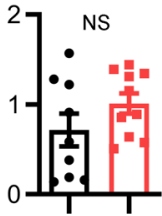

K

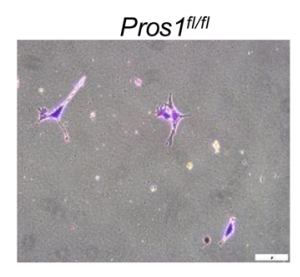

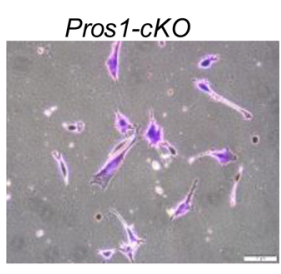

$\mathbf{L}$

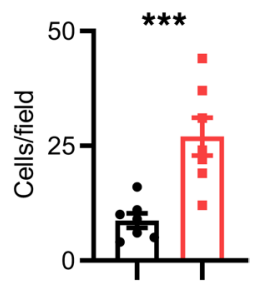

Figure 6. Conditioned medium from Pros1-ablated BMDMs enhances Lewis lung carcinoma cancer cell aggressiveness. Lewis lung carcinoma (LLC) cells were educated ( 24 hours) by the indicated conditioned medium (CM) and subject to a battery of in vitro assays. (A and B) Colony survival after 10 days. (A) Representative images (number of cells plated/well is indicated) and (B) quantification of surviving colonies. The mean $\pm S E M ; n=6$ mice/group. ${ }^{* * * *} P<$ $0.0001 ; 2$-way ANOVA. (C) Representative images and (D) quantification of soft agar colonies (arrows) after 21 days. Scale bars: $50 \mu \mathrm{m}$. Average \pm SEM; $n=$ 8 mice/group. ${ }^{* *} P=0.002$; $t$ test. (E) Representative Western blots and (F) quantifications showing ERK and AKT activation from educated LLC cells. $n=8$ mice/group; 3 independent experiments. The average ratio \pm SEM is plotted. ${ }^{*} P=0.018,{ }^{* *} P=0.006$; $t$ test. Representative Western blot (G) and quantifications (H) for MMP9 protein in lungs of metastatic mice. $n=7-8$ mice/group in 2 independent experiments. The average ratio \pm SEM is plotted. ${ }^{* * *} P=0.001$; $t$ test. A representative Western blot (I) and quantifications (J) showing E-cadherin, $\mathrm{N}$-cadherin, and vimentin in educated LLC cells. $n=9-10$ mice/group in 3 independent experiments. The average ratio $\pm \mathrm{SEM}$ is plotted. ${ }^{*} P=0.04,{ }^{* *} P=0.009$; $t$ test. (K and $\mathbf{L}$ ) Invasion assay: Representative images (K) and quantifications (L) of cells that invaded through Matrigel after education with the indicated CM. Scale bars: $50 \mu \mathrm{m}$. (L) The average cell number \pm SEM of invaded cells is shown from 7-10 different fields, $n=7$ mice/group in 3 independent experiments. ${ }^{* *} P=0.0007 ; t$ test. NS, nonsignificant.

in primary tumor growth (Supplemental Figure 5, C and D). Conversely, LLC cells educated by CM-BMDM-cKO from tumor-bearing mice - but not CM-BMDM-fl/fl - produced significantly larger metastases $\left(1.1 \pm 0.15 \mathrm{~mm}^{2}\right.$ versus $0.42 \pm 0.09 \mathrm{~mm}^{2}$; Figure 5 , A and B). No significant effect was seen on metastasis numbers, although a trend toward increased metastases was observed for CM-BMDM-cKO from tumor-bearing mice (Figure 5C). Noticeably, BMDMs isolated from tumor-bearing mice induced an overall higher metastatic burden (Figure 5, A-C), demonstrating a bidirectional cross-talk between LLC and host macrophages, with PROS1 being a molecular switch of metastasis.

To elucidate whether PROS1 correspondingly functions as a molecular metastatic switch in other cancers, GFP-AT3 cells were educated (96 hours) and s.c. injected into WT mice. When injected, AT3 cells have poor metastatic potential, as confirmed by our experiment: 4 of 8 mice educated with CM-BMDM-fl/fl developed metastasis, which were relatively small in area $(0.03 \pm$ $0.01 \mathrm{~mm}^{2}$ ). Remarkably, 7 of 8 mice that were injected with cells educated by CM-BMDM-cKO developed larger $\left(0.1 \pm 0.02 \mathrm{~mm}^{2}\right)$ and more numerous $(2.5 \pm 0.6$ versus $0.87 \pm 0.4$; Figure $5, D-F)$ lung metastases. This was also observed superficially by their GFP fluorescence and GFP protein levels in lung lysates (Figure 5, G and $\mathrm{H})$. Akin to the LLC model, primary tumor growth was not affected in AT3 mammary breast cancer (Supplemental Figure 5, E and F). Taken together, using two cancer models, our results show that PROS1 is a potential modulator in host macrophages, its loss shifting their cytokine secretion toward a proinflammatory profile, which in turn exacerbated lung and mammary tumor metastasis. 
A
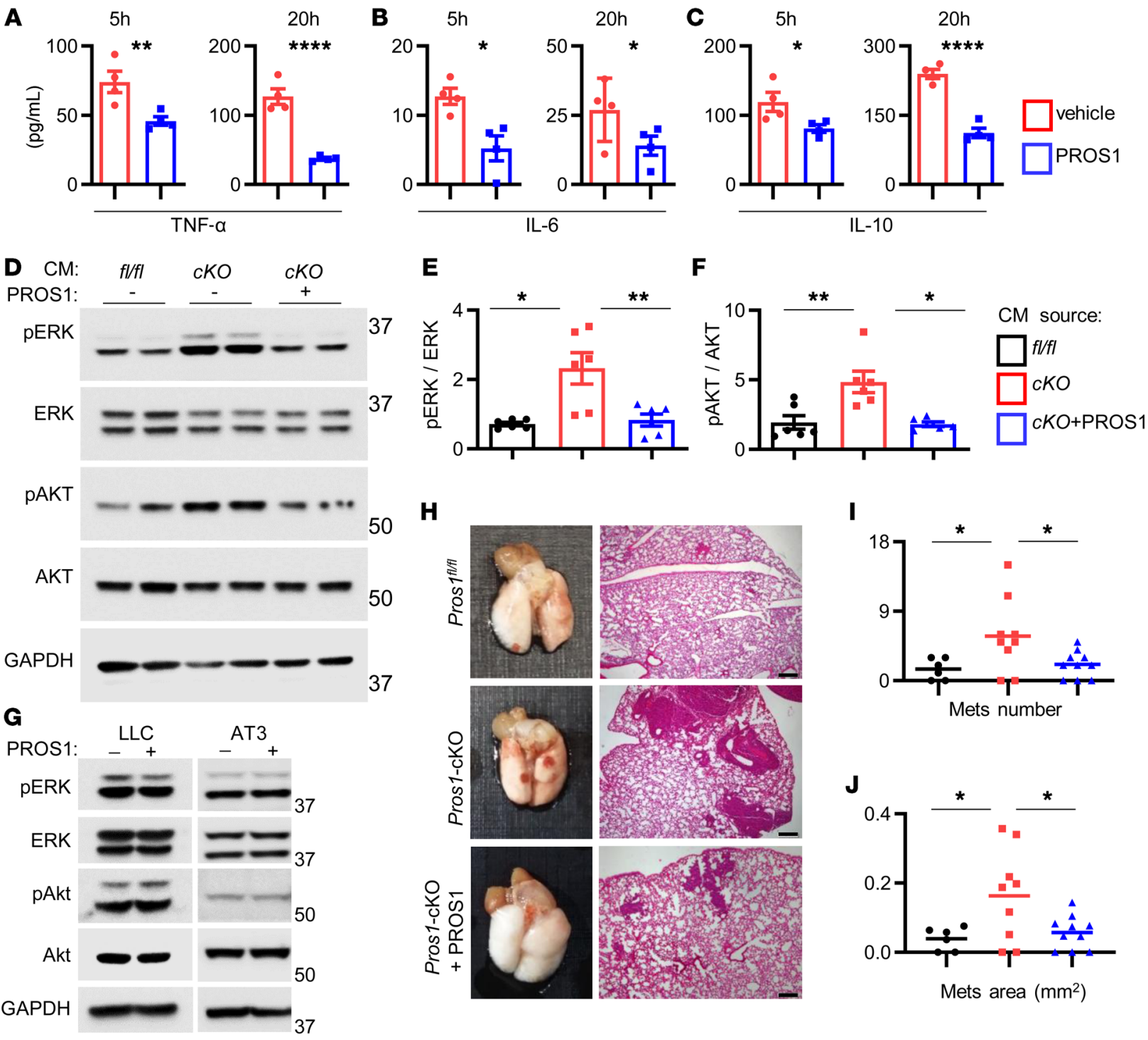

50

H 50
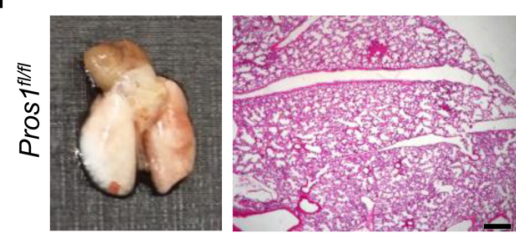

I
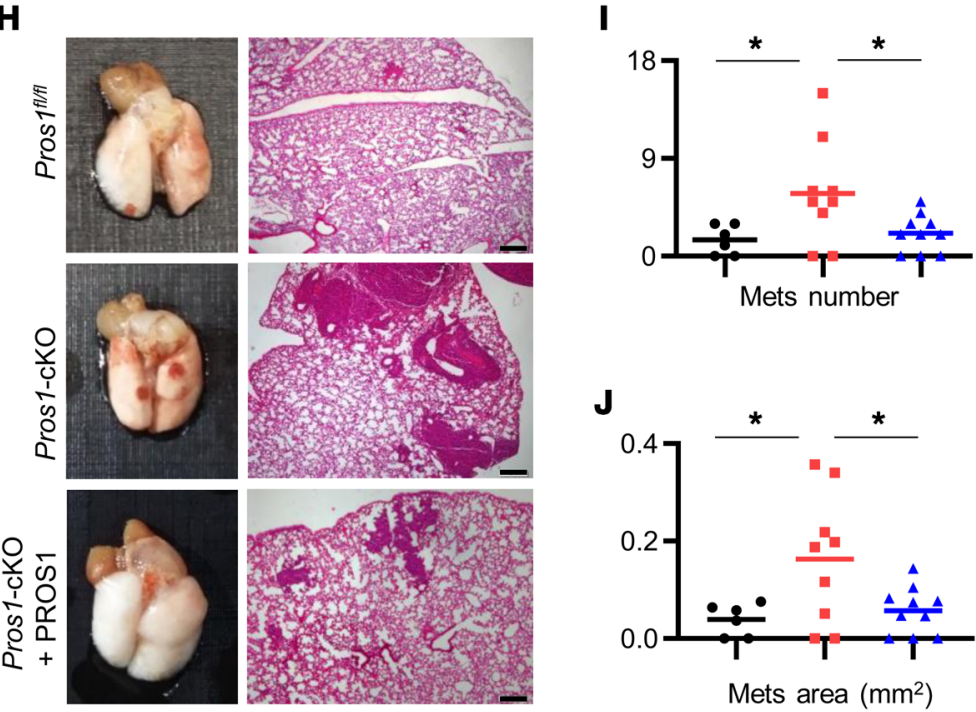

J
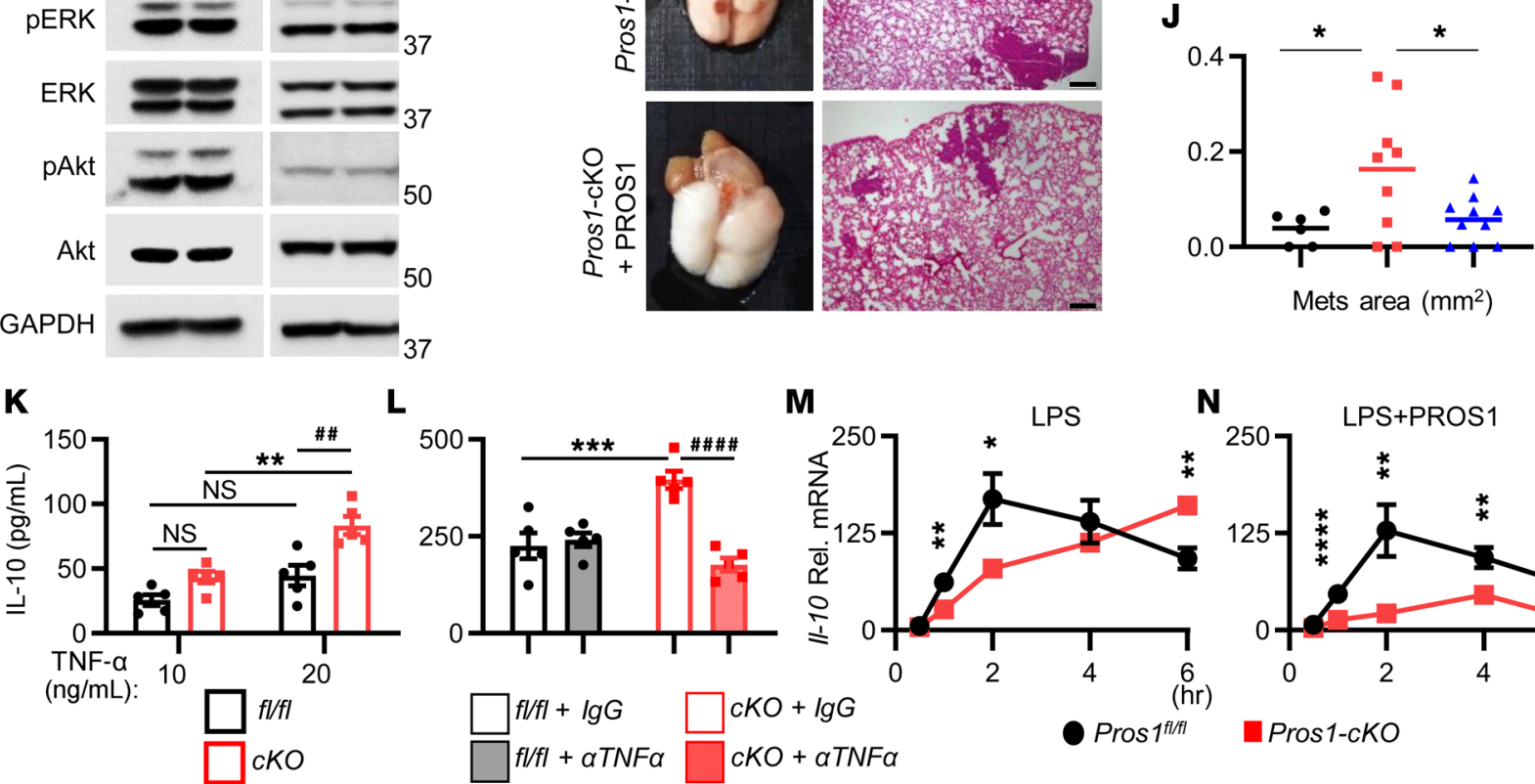

L

M
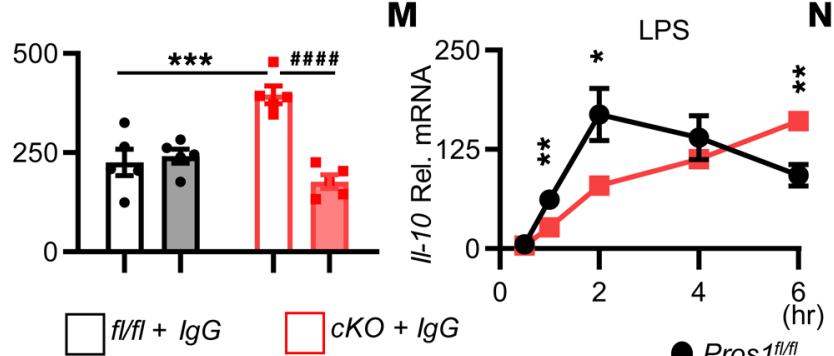

$\mathbf{N}$

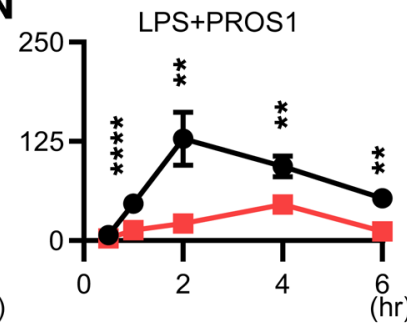

$\square f l / f l+\lg G \quad \square c K O+\lg G$
$\square f l / f l+\alpha T N F \alpha \square c K O+\alpha T N F \alpha$

Prosifi/

-Pros1-cKO

Figure 7. Exogenous PROS1 dampens the inflammatory signature of Pros1-cKO BMDMs and mitigates metastasis in vivo. (A-C) Cytokine secretion by LPS-stimulated (100 ng $/ \mathrm{mL}$ ) tumor-naive Pros1-cKO BMDMs, incubated with vehicle or PROS1 (25 nM). ELISA measurements at 5, 20 hours. Mean \pm SEM; $n=4 / g r o u p$. TNF- $\alpha$ $\left(\mathbf{A},{ }^{* *} P=0.007 ;{ }^{* * *} P=0.0001\right), \mathrm{IL}-6\left(\mathbf{B},{ }^{*} P=0.03 ; 0.05\right), \mathrm{IL}-10\left(\mathbf{C},{ }^{*} P=0.02 ;{ }^{* * * *} P<0.0001\right) ; t$ test. (D-F) PROS1 reverses the prometastatic potential of cKO-BMDMs. Representative Western blot (D), quantification of ERK (E; $\left.{ }^{*} P=0.03 ;{ }^{* *} P=0.01\right)$, and AKT (F; ${ }^{*} P=0.02 ;{ }^{* *} P=0.003$ ) activation in LLC cells educated with CM from $\mathrm{fl} / \mathrm{fl}$ or $\mathrm{CKO}$ cells, with or without PROS1 (25 nM). Two mice of 6 are shown/group; 1-way ANOVA. (G) Western blot analysis showed no direct activation of AKT or ERK by PROS1-treated LLC cells ( $25 \mathrm{nM}, 24$ hours). A representative blot of 3 experiments is shown. (H-J) PROS1 suppressed the metastasis-inducing potential of cKOBMDMs in vivo. (H) WT mice ( $n=6-10 /$ group) were inoculated with educated cells as described in $\mathbf{D}$. Lungs were assessed for metastases 3 weeks later (left). $\mathrm{H} \& \mathrm{E}$ sections (right) were used to quantify the average metastatic area $\left(\mathbf{l} ;{ }^{*} P=0.03\right)$ and number $\left(\mathbf{j} ;{ }^{*} P=0.04\right) ; 1$-way ANOVA. The horizontal line marks the mean tumor weight. Scale bars: $200 \mu \mathrm{m}$. (K and L) TNF- $\alpha$ regulates IL-10 secretion in cKO BMDMs. (K) ELISA measurements of IL-10 secretion by TNF- $\alpha$-stimulated (10 and 20 ng/ $\mathrm{mL} ; 24$ hours) control and cKO BMDMs. Mean $\pm \mathrm{SEM} ; n=5$ mice/group. ${ }^{* *} P=0.0014$; ${ }^{\#} P=0.002$; NS, nonsignificant; 2-way ANOVA. (L) ELISA measurements of IL-10 secretion from LPS-stimulated ( $100 \mathrm{ng} / \mathrm{mL} ; 24$ hours) $\mathrm{fl} / \mathrm{fl}$ or cKO pretreated with $\lg \mathrm{G}$ or anti-TNF- $\alpha$ neutralizing antibody. Mean $\pm \mathrm{SEM} ; n=5$. ${ }^{* * *} P=0.0006$; \#\#\#\# $<0.0001 ; 2$-way ANOVA. (M and $\mathbf{N}$ ) Relative $I-10$ mRNA levels of LPS-simulated (100 ng/mL) BMDMs without $\left(\mathbf{M} ;{ }^{*} P=0.02,{ }^{* *} P<0.01[=0.002\right.$ and 0.004 at 1 and 6 hours, respectively] or with PROS1 (25 nM) $\left(\mathbf{N} ;{ }^{* *} P \leq 0.01,{ }^{* * *} P<0.0001\right)$. Relative mean \pm SEM; $n=5$ mice/group; Holm-Sidak multiple $t$ test. 
CM of PROS1-deficient macrophages drives multiple invasive properties of tumor cells. We next characterized the specific cellular phenotypes inflicted by CM-BMDM-cKO underlying the elevated metastatic burden observed in Pros1-cKO mice. LLC cells educated by $\mathrm{CM}$ from tumor-naive BMDM-cKO cultures had more surviving colonies after 10 days (Figure 6, A and B) and superior growth in soft agar (Figure 6, C and D). Increased activation of oncogenic and survival pathways was observed, as indicated by elevated pERK and pAKT levels in LLC cells educated by CM-BMDM-cKO (Figure 6, E and F). Similar effects were observed for AT3 cells, with the exception of unaffected pERK (Supplemental Figure 6, A-E).

To gain insight into the metastasis-promoting molecular changes within tumor cells induced by CM-BMDM-cKO, we examined expression of MMP9, a potent extracellular matrix-degrading (ECM-degrading) enzyme known to facilitate metastasis of many tumors, including lung cancer $(45,46)$. Although MMP9 levels in metastatic lungs of Prosi $1^{f / f l}$ mice were barely detectable, MMP9 expression was highly upregulated in metastatic cKO lungs (Figure 6, G and $\mathrm{H}$ ). We further examined markers associated with the EMT-supporting migratory and invasive properties. We observed downregulation of E-cadherin and upregulation of $\mathrm{N}$-cadherin in cells educated with CM-BMDM-cKO but not CM-BMDM-fl/fl, indicating cells underwent EMT. No changes were recorded for vimentin (Figure 6, I and J). Moreover, this EMT-related molecular switch was reflected phenotypically, in that LLC educated by CM-BMDMcKO had superior invasive potential (Figure 6, K and L).

PROS1 supplementation suppresses the proinflammatory profile of macrophages and restrains their prometastatic properties. Our data indicate that the proinflammatory nature of Pros1-deficient macrophages supported a metastatic outcome. If so, we reasoned that adding exogenous PROS1 would dampen the elevated inflammation of Pros1-cKO BMDMs. Indeed, addition of purified PROS1 (25 nM) to LPS-stimulated BMDM-cKO reduced secretion of TNF- $\alpha$, IL-6, and IL-10 after 5 hours, which persisted after 20 hours (Figure 7, A-C). We next tested whether exogenous PROS1 would rescue the aggressive phenotype of LLC cells acquired during education with CM-BMDM-cKO. Remarkably, addition of exogenous PROS1 to cKO-BMDMs attenuated phosphorylation of both ERK and AKT in educated LLC cells (Figure 7, D-F). Importantly, purified PROS1 directly added to LLC or AT3 cells did not affect pERK or pAKT signaling, indicating that diminished ERK and AKT phosphorylation was not due to a direct effect of PROS1 carried over from the CM onto cancer cells (Figure 7G). The reversal of oncogenic signaling in LLC cells after PROS1 rescue was further extended in vivo (Figure 7, $\mathrm{H}-\mathrm{J}$ ). PROS1 addition reversed the number and size of metastatic nodules (Figure 7, I and J). Altogether, our results show that addition of PROS1 was sufficient to inhibit inflammation in Pros1-cKO BMDMs, and to obliterate their prometastatic effect in vivo through modulation of ERK and AKT oncogenic pathways.

To gain further mechanistic insight into the factors promoting metastasis secreted by cKO BMDMs, we focused on IL-10, which was prominently upregulated after Pros 1 inhibition (Figure 2, A and B, Supplemental Figure 2, F and G, Figure 3, C and H, and Figure 4, A and B). We first determined whether IL-10 levels are linked with the inflamed state observed in BMDM-cKO cells. IL-10 production was measured by ELISA after TNF- $\alpha$ stimula- tion. Higher TNF- $\alpha$ levels induced higher IL-10 secretion by both control and cKO BMDMs, with a stronger response in cKO cells (Figure 7K). Akin to TNF- $\alpha$, LPS treatment also increased IL-10 secretion, which was inhibited in the presence of anti-TNF- $\alpha$ neutralizing antibodies (Figure 7L). Furthermore, the dynamics of $\mathrm{Il}$ 10 mRNA after LPS stimulation differed between control and cKO BMDMs. An initial peak at 2 hours after stimulation was recorded for control and cKO cells, yet IL-10 expression was suppressed in control BMDMs, but continued to rise in Pros1-deficient cells, indicating the lack of a PROS1-dependent regulatory mechanism (Figure 7M). Notably, this elevation in IL-10 expression was reversed in the presence of purified PROS1 (Figure 7N). Thus, IL-10 expression within BMDMs could be gauged by PROS1 levels and its upregulation in $\mathrm{CKO}$ BMDMs was TNF- $\alpha$ dependent and linked to the inflammatory status of the cells.

The prometastatic potential of Pros1-cKO BMDMs is largely mediated by $I L-10$. High IL-10 expression by tumor-infiltrating macrophages was shown to correlate with advanced stages of non-small cell lung cancer and associated with poor prognosis (47). We therefore hypothesized that the prometastatic nature of cKO macrophages may be mediated via IL-10. IL-10 neutralization during the education period significantly suppressed the survival and invasive potential of LLC cells (Figure 8, A-D). Moreover, IL-10 blockade suppressed the phosphorylation of ERK, AKT, and STAT3 in LLC cells educated by CM-BMDM-cKO (Figure 8, $\mathrm{E}$ and F). IL-10 neutralization also arrested the metastatic potential of LLC cells induced by education with CM-BMDM-cKO without affecting CM-BMDM-fl/fl-educated cells. These effects were characterized by reduction of metastatic area from $0.38 \pm$ $0.09 \mathrm{~mm}^{2}$ for IgG-treated cKO cells to $0.087 \pm 0.05 \mathrm{~mm}^{2}$ in the presence of anti-IL-10. Similarly, the number of metastases was reduced from $15.2 \pm 3.1$ for IgG-treated cKO cells to $4 \pm 1.8$ in the presence of anti-IL-10 (Figure 8, G-I). Taken together, our results show that the absence of PROS1 in macrophages led to an elevated proinflammatory cytokine profile, including high TNF- $\alpha$ levels, which in turn stimulated IL-10 production. IL-10 increased activation of ERK, AKT, and STAT3 signaling, promoting survival and invasive phenotypes observed in vitro and in vivo.

Secretion of TNF- $\alpha$ and IL-6 by macrophages is regulated by PROS1 through TAMs and diverges from that of IL-10. We previously showed that PROS1 is a cognate ligand for the TAMs (42) and that PROS1 inhibits inflammation in a murine peritonitis model (16). Similarly, MERTK regulates inflammation by inhibiting TNF- $\alpha$ secretion in macrophages $(17,48)$. We next tested whether the effects of PROS1 in BMDMs are mediated by MERTK. At steady state, phosphorylation of MERTK (pMERTK) in cKO BMDMs was only $42 \%$ of that in PROS1-expressing cells (Supplemental Figure 7, A and B), indicating that endogenous PROS1 is a MERTK agonist. Addition of PROS1 to starved BMDMs increased pMERTK in BMDM-fl/fl and BMDM-cKO cultures, validating PROS1 as a MERTK agonist in BMDMs (Supplemental Figure 7, C and D). Of note, addition of PROS1 to BMDM-fl/fl stimulated pMERTK to a greater extent than in BMDM-cKO (Supplemental Figure 7, $\mathrm{C}$ and D), supporting endogenous PROS1 as a major agonist of MERTK in macrophages. Next, once verified (Supplemental Figure 7, E and F), TAM kinase inhibitors were added to BMDM cultures to identify whether PROS1 effects are mediated via TAMs. Akin to 
A

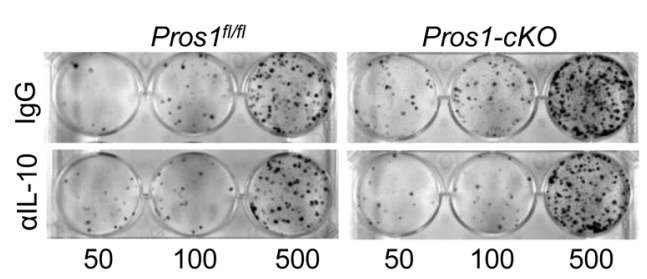

C
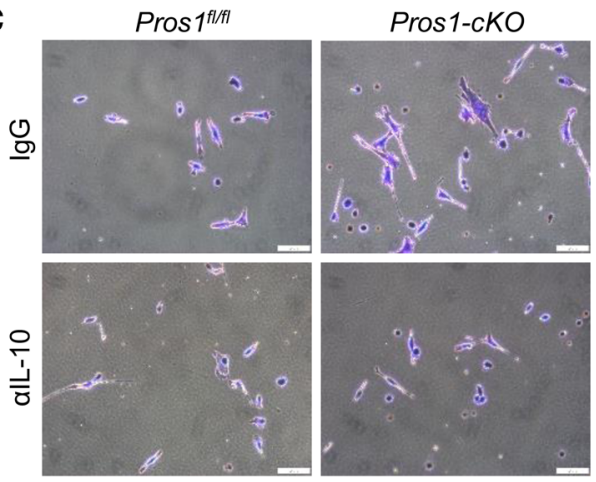

E

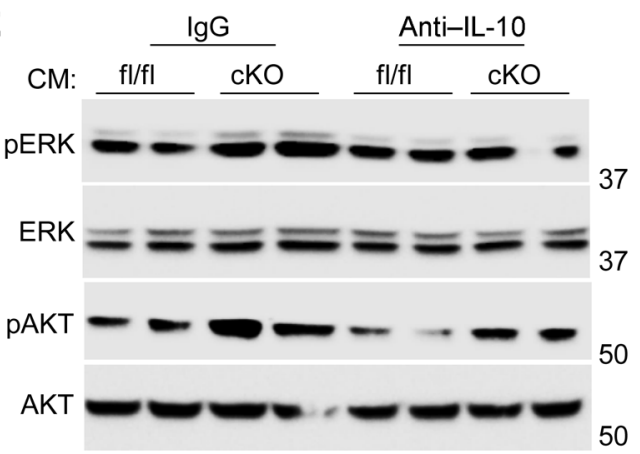

pSTAT3

STAT3 $=-m=-m=-m$

G

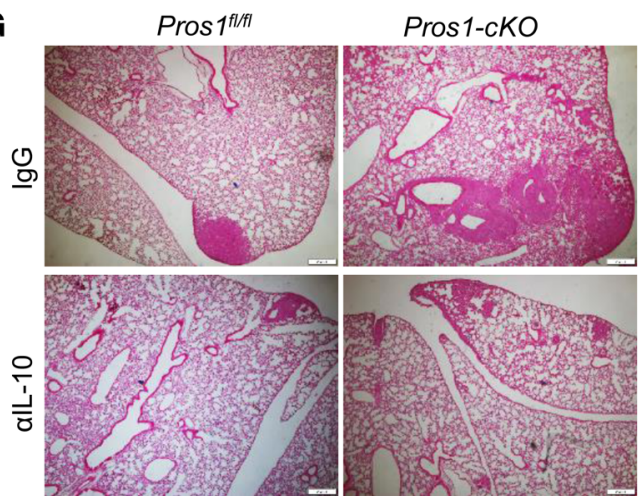

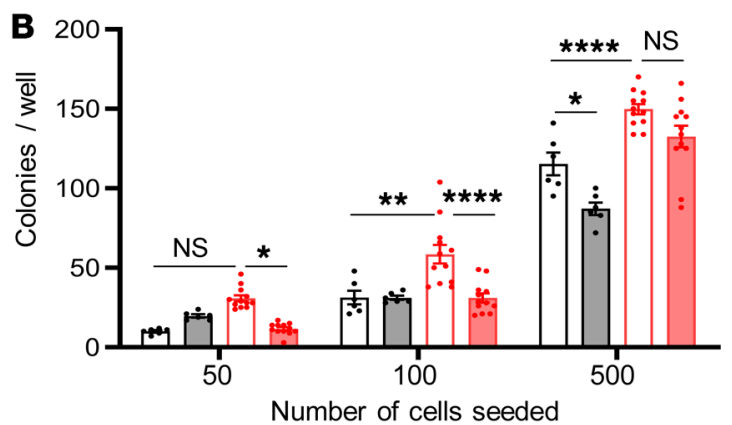

D

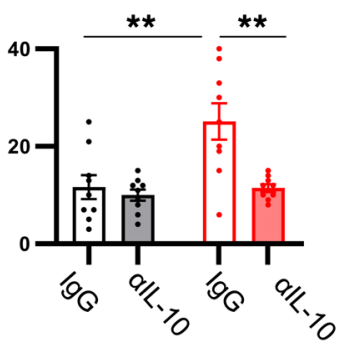

CM:

$\mathbf{F}$

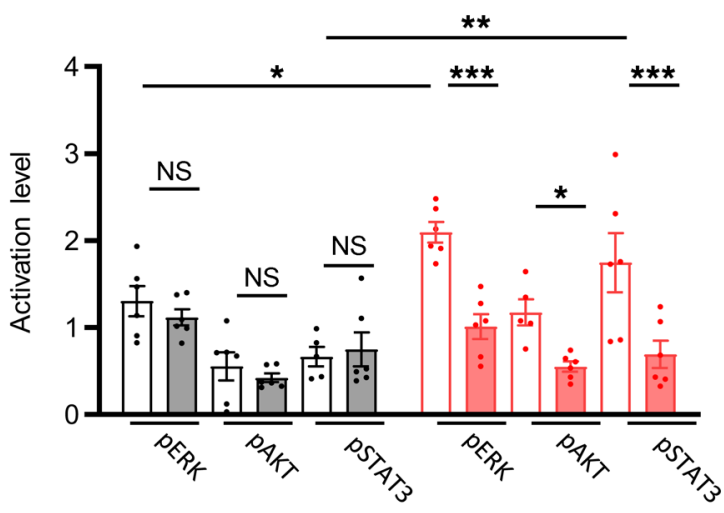

H

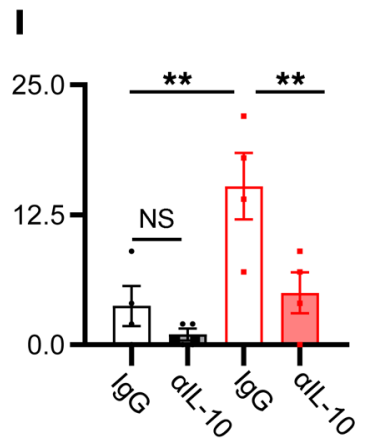

Mets area $\left(\mathrm{mm}^{2}\right)$

Mets number 
Figure 8. Neutralization of IL-10 in Pros1-cKO conditioned medium reduces Lewis lung carcinoma aggressiveness and suppresses in vivo metastasis. Lewis lung carcinoma (LLC) cells were educated by the indicated CM with control (IgG) or anti-IL-10 neutralizing antibodies ( $\alpha$ IL-10) for 24 hours and subject to a battery of in vitro assays. (A and $\mathbf{B}$ ) Colony survival after 10 days. Representative images of educated cells plated at different densities as indicated (A) and quantification of colonies (B). Mean \pm SEM; $n=6-12$ mice/group; 3 independent experiments. ${ }^{*} P=0.02 ;{ }^{*} P=$ 0.004 ; ${ }^{* * *} P<0.0001 ; 2$-way ANOVA. (C and D) Matrigel invasion assay. Representative images (C) and quantification (D) of educated cells that traversed the ECM-coated membrane without or with IL-10 neutralization. Scale bars: $50 \mu \mathrm{m}$. (D) The average cell number from 7-9 different fields \pm SEM is shown, 4-5 mice/group, 2 independent experiments. ${ }^{*} P=0.0017$ (left) and 0.0014 (right); 2-way ANOVA. (E) Representative Western blots and quantifications (F) of ERK, AKT, and STAT3 activation from educated LLC cell lysates. Band intensities were calculated from 5-6 mice/group, 2 independent experiments. The average ratio (phosphorylated/total) $\pm \mathrm{SEM}$ is plotted. ${ }^{*} P=0.04$ (ERK), ${ }^{* *} P=0.002$ (STAT3) for control versus $\mathrm{CKO}$ with IgG; and ${ }^{*} P=0.05$ (AKT), ${ }^{* *} P=0.001$ (ERK and STAT3) for cKO with IgG versus cKO with $\alpha$ IL-10; 2-way ANOVA. (G-I) Educated LLC cells described above were s.c. injected into the flank of WT mice and 3 weeks later lung metastasis was evaluated. (C) Representative images of H\&E-stained sections from lungs. Scale bars: $200 \mu \mathrm{m}$. (H and I) Scoring of lung metastases (Mets), the average metastatic area $(\mathbf{H})$ and number $(\mathbf{I}) \pm$ SEM are shown. ${ }^{*} P=0.04$ and 0.02 for $\mathbf{H}$ (left and right, respectively); ${ }^{*} P=0.01$ and 0.008 for I (left and right, respectively); 2-way ANOVA. NS, nonsignificant.

PROS1 reversing metastasis in vivo (Figure 7, $\mathrm{H}-\mathrm{J}$ ), the potential of CM-BMDM-cKO to support in vitro invasion through Matrigel was abrogated in the presence of PROS1 (Figure 9, A and B). To establish that PROS1 signaling is mediated by TAMs, the small molecule pan-TAM inhibitor UNC4241 was used. Surprisingly, UNC4241 did not interfere with the capacity of PROS1 to suppress invasion (Figure 9, A and B). Given that IL-10 within CM-BMDMcKO promotes invasion (Figure 8, C and D), we inspected the regulation of IL-10, TNF- $\alpha$, and IL- 6 by TAMs. Whereas both UNC4241 (pan-TAM) and UNC4203 (MERTK specific) inhibitors abated the capacity of PROS1 to inhibit TNF- $\alpha$ and IL- 6 secretion, IL-10 levels were unaffected (Figure 9C). These results suggest that although PROS1 suppression of TNF- $\alpha$ and IL- 6 depends on the kinase function of MERTK, the regulation of IL-10 by PROS1 may be regulated either by a nonkinase function of MERTK or in an altogether MER-independent manner. Moreover, these results agree with the invasive property of LLC being IL-10 dependent and the incapacity of UNC4241 to interfere with PROS1 inhibition of LLC invasion (Figure 9, A and B). STAT1 and SOCS3 are known downstream effectors of MERTK in various cells $(14,49)$. Both pSTAT1 levels and Socs 3 transcription increased after PROS1 stimulation, as expected. However, addition of UNC4203 was unable to perturb pSTAT1 or Socs3 stimulation by PROS1 (Figure 9, D-F). Taken together, our data indicate that in macrophages, TNF- $\alpha$ and IL-6 were mainly regulated by MERTK kinase activity, and the data provide evidence for the divergence of IL-10 regulation.

PROS1 ablation in myeloid cells abrogates their T cell stimulatory potential. We next sought to identify whether deletion of PROS1 in myeloid cells has a broader systemic effect involving additional immune cells, as immune-based antitumor responses within the primary and metastatic sites are known to shape tumor progression and metastases $(30,50)$. IL-10 secreted by macrophages elicits immune suppression by inhibition of $\mathrm{T}$ cell antitumor responses $(51,52)$. We therefore reasoned that elevated secretion of IL-10 by cKO BMDMs and lung macrophages might support metastasis through immune suppression. We first tested the effect of macrophage-derived PROS1 on T cell proliferation within a metastatic context. For this, stimulated T cells were incubated in the presence of control or Pros1-cKO macrophages sorted from metastatic lungs. Compared with control macrophages, PROS1-deficient macrophages significantly inhibited $\mathrm{T}$ cell proliferation by $23 \%$, revealing that $\mathrm{cKO}$ macrophages suppressed $\mathrm{T}$ cell proliferation. Addition of purified PROS1 to the culture significantly rescued this suppressive capacity, suggesting a $\mathrm{T}$ cell stimulatory function for PROS1 (Figure 10A and Supplemental Figure 8B). Furthermore, IL-10 neutralizing antibodies abrogated the inhibitory effect of PROS1-deficient macrophages on $\mathrm{T}$ cell proliferation (Supplemental Figure 8, A and B), pointing to an immune-suppressive role for IL-10 after PROS1 ablation in metastatic lung macrophages.

Examination of total $\left(\mathrm{CD}^{+}\right) \mathrm{T}$ cells from metastatic lungs revealed a trend toward decreased $\mathrm{T}$ cell numbers in cKO lungs, but similar $\mathrm{CD}^{+}$and $\mathrm{CD} 8^{+}$frequencies in both mouse models (Figure 10, B and C). NO produced by macrophages is upregulated by IL-10 (53) and was shown to inhibit T cell activation $(54,55)$. We measured elevated nitrite levels (the product of NO decomposition) in the medium of Pros1-cKO macrophages sorted from metastatic lungs, which decreased upon IL-10 neutralization (Figure 10D). We further tested the levels of the $\mathrm{T}$ cell receptor $\zeta$-chain subunit ( $\zeta$-chain) relative to $\mathrm{CD} 3 \varepsilon$, which serves as a biomarker for an immunosuppressive environment, directly gauging $\mathrm{T}$ cell function (56). Compared with PROS1-proficient mice, expression of $\zeta$-chain was significantly downregulated in $\mathrm{T}$ cells from cKO metastatic lungs, indicating their impaired function in a metastatic milieu (Figure 10, $\mathrm{E}$ and $\mathrm{F}$ ). In line with reduced $\zeta$-chain expression, $\mathrm{CD}^{+}$and $\mathrm{CD} 4^{+} \mathrm{T}$ cells isolated from cKO mice had higher levels of the immune-suppressive molecules programmed cell death protein 1 (PD1) and cytotoxic T lymphocyte-associated protein 4 (CTLA-4), respectively (Figure 10, G-J), insinuating induced T cell immunosuppression in metastatic lungs of cKO mice. Collectively, our data revealed that PROS1 stimulates $\mathrm{T}$ cell proliferation directly and indirectly by inhibition of IL-10 production within macrophages. Additionally, reduced $\zeta$-chain and elevated immune checkpoint inhibitors indicate effects on T cell activation after a myeloid-specific PROS1 deletion within the metastatic niche.

Our results pointing at a possible immunosuppressive state of T cells in Pros1-cKO tumor-bearing mice could also be due to suboptimal stimulation by DCs, given that tumor-infiltrating DCs expressing CD11 $\mathrm{c}^{+} \mathrm{MHC}-\mathrm{II}^{+}$limit tumor progression through T cell activation (57). To test whether PROS1-cKO DCs possess decreased $\mathrm{T}$ cell stimulatory potential, we generated BMDCs from control and $\mathrm{cKO}$ mice. Compared with BMDMs, LysM-Cre efficiency was reported to be less effective in BMDCs, reaching 31\% (22). The overall knockout efficiency in our BMDC culture reached $47 \%$, yet in stark contrast to BMDMs, no changes were reported for tumor-modulating cytokines, such as $T g f b$, Ifng, Gmcsf, Il-12p35, and Il-10 (Figure 11A). Although similar frequencies of $\mathrm{CD} 45^{+} \mathrm{CD} 11 \mathrm{c}^{+}$cells were obtained, the BMDC population expressing $\mathrm{MHC}-\mathrm{II}^{\mathrm{hi}}$ was significantly reduced in 
A

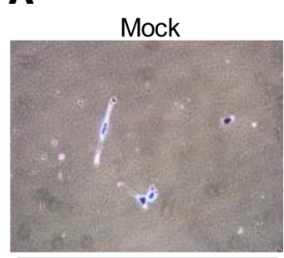

Pros $1^{1 / f / 1}$
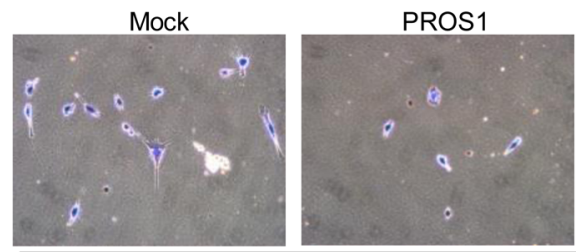

Pros1-cKO

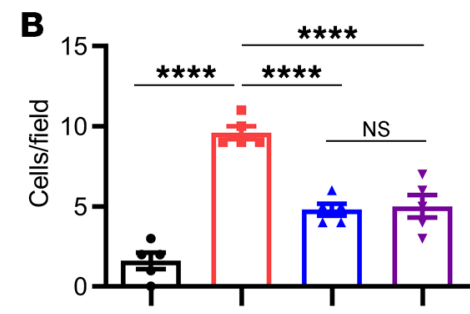

CM source:

$\square$ fl/fl Mock $\square$ cKO+PROS1

$\square$ cKO Mock $\square$ cKO+PROS1+UNC4241

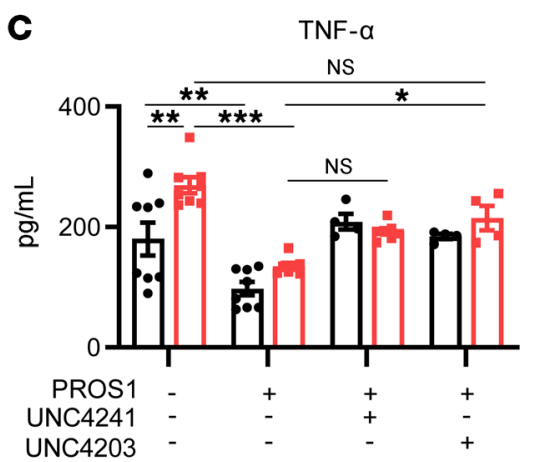

D

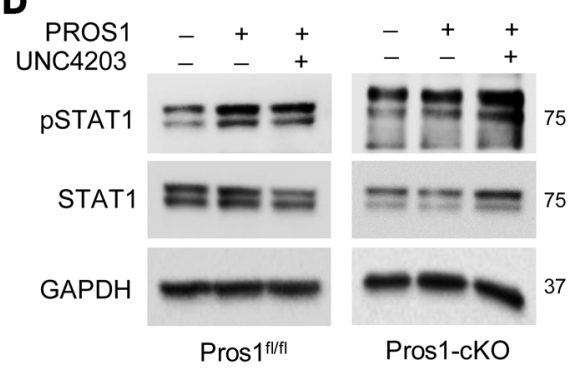

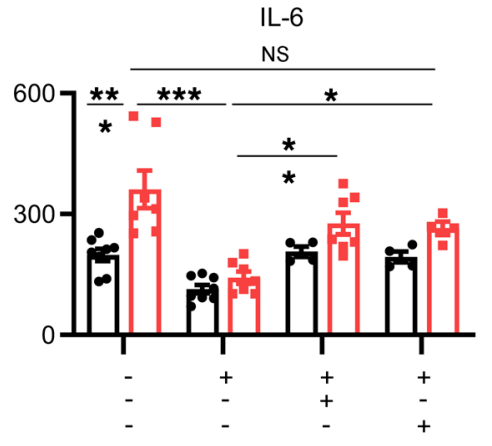

$\mathbf{E}$

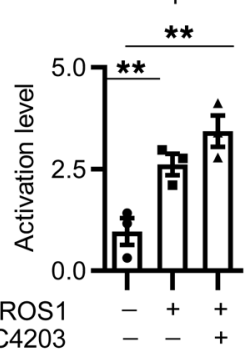

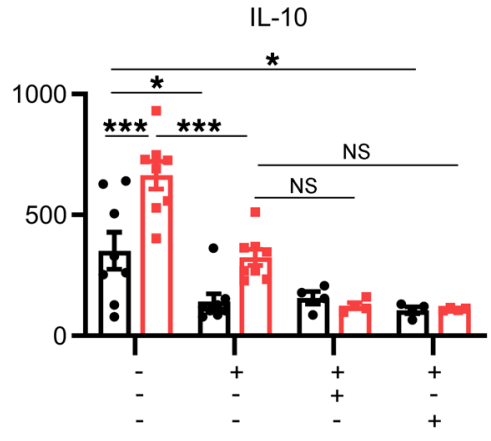

$\mathbf{F}$

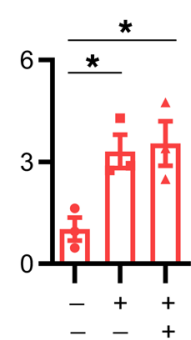

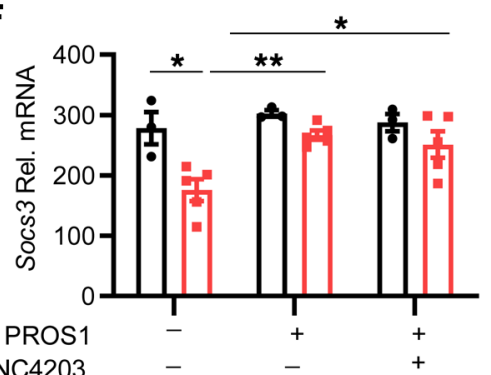

Figure 9. Distinct requirements for the MERTK kinase activity in PROS1-mediated regulation of TNF- $\alpha$ and IL-6 versus IL-10. (A and B) Matrigel invasion assay. LLC cells were educated with BMDM-CM from fl/fl BMDMs or cKO BMDMs (mock), or supplemented with PROS1 or with PROS1 + UNC4241 (pan-TAM kinase inhibitor). Representative images (A) and quantifications (B) of cells that invaded through Matrigel. Graphs present the average cell numbers from 7-10 different fields \pm SEM, 4-5 mice/group, 2 independent experiments. ${ }^{* * * *} P \leq 0.0001$; 1-way ANOVA. (C) ELISA measurements of secreted cytokines in CM of control and cKO BMDMs. Serum-starved BMDMs were treated with PROS1 (25 nM) alone or with 500 nM UNC4241 (pan-TAM) or UNC4203 (MER-specific) inhibitors for 1 hour, and then stimulated with LPS for 24 hours. Average values \pm SEM are shown for $4-8$ mice, 2 independent experiments. TNF- $\alpha$ : $P=0.05$, ${ }^{* *} P \leq 0.005,{ }^{* *} P=0.001 ; \mathrm{IL}-6:{ }^{*} P \leq 0.05,{ }^{* *} P=0.005,{ }^{* * *} P \leq 0.0004 ; \mathrm{IL}-10:{ }^{*} P \leq 0.05,{ }^{* *} P \leq 0.0004 ; 2$-way ANOVA. (D-F) MERTK kinase activity is not essential for STAT1 activation by PROS1. Representative Western blots (D) and quantification (E) of STAT1 activation in LPS-stimulated BMDMs either without or with PROS1 and UNC4203 (500 nM), as indicated. GAPDH served as a loading control. Graphs present the average values \pm SEM, 6 mice/group; 2 independent experiments. Each symbol represents a pool of 2 mice/group. ${ }^{*} P \leq 0.012$ and ${ }^{*} P \leq 0.01$ for cKO and control, respectively; 1 -way ANOVA. (F) Socs3 relative mRNA levels from BMDMs described in $\mathbf{D}$. Mean $\pm \mathrm{SEM} ; n=6-10$ mice; each symbol represents a pool of 2 mice. * $P \leq 0.05 ; 2$-way ANOVA.

cKO mice (Figure 11, B and C). Moreover, CD11 ${ }^{+}$MHC-II ${ }^{\text {hi }}$ cells expressed less of the CD40 costimulatory molecule (Figure 11, $\mathrm{D}$ and $\mathrm{E}$ ), suggesting their impaired ability to stimulate $\mathrm{T}$ cells. Analyzing DCs from metastatic lungs (Figure 11, F-I), we found a trend toward a reduction in the $\mathrm{CD} 11 \mathrm{c}^{+} \mathrm{MHC}-\mathrm{II}^{\mathrm{hi}}$ population in cKO lungs (Figure 11, F and G), suggesting their attenuated potential to stimulate $\mathrm{T}$ cells in situ. This is supported by their reduced expression of CD40 and CD86 costimulatory molecules (Figure 11, H and I), implying impaired $\mathrm{T}$ cell stimulatory potential in a metastatic environment. Taken together, our results indicate that inhibition of PROS1 in myeloid cells leads to a broad immune-suppressive phenotype involving macro- phages, DCs, and T cells, with PROS1 acting both as a regulator of inflammation within macrophages and affecting $\mathrm{T}$ cell proliferation directly and indirectly via suppression of IL-10.

\section{Discussion}

$\mathrm{BM}$-derived immune cells contribute to tumor angiogenesis, invasion, and metastasis $(2,3,30,58,59)$, but the signaling mechanisms underlying these processes have not been fully elucidated. The metastasis-promoting functions of immune cells were shown to be driven by numerous molecules, some of which are FLT-1 (VEGF-R1), CARD9, and MMP9 (60-63). Yet, macrophage-derived proteins possessing antimetastatic qualities are sparse. Here, 

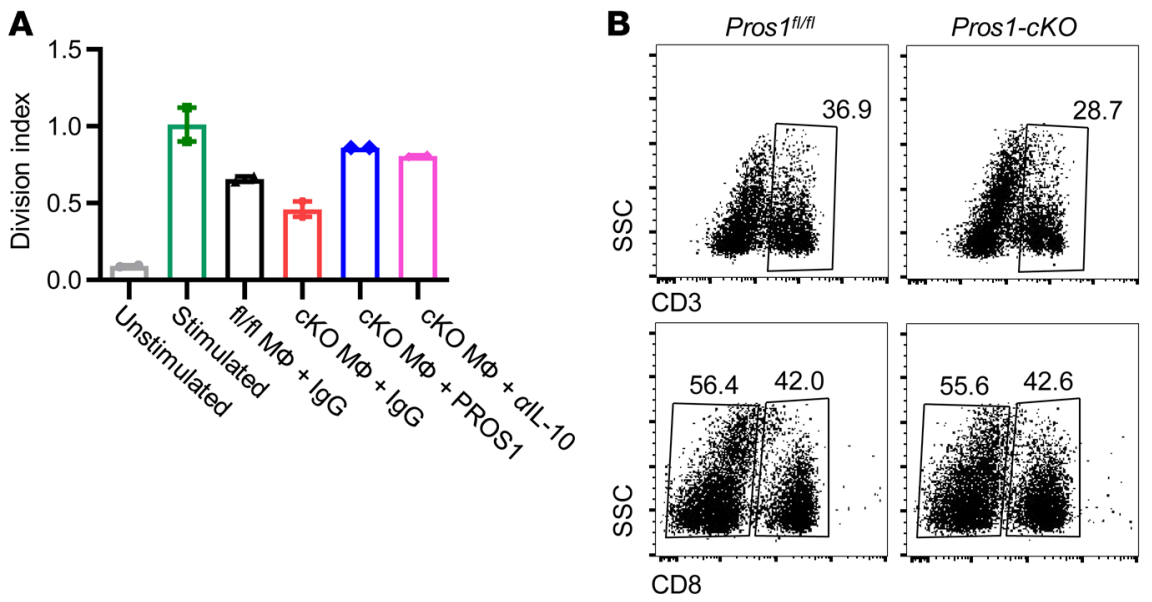

E

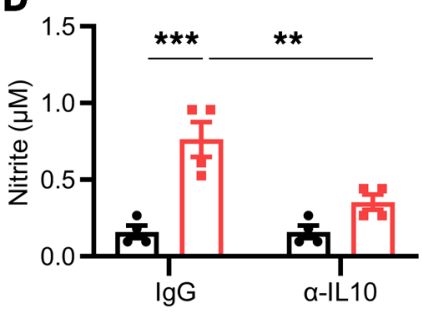

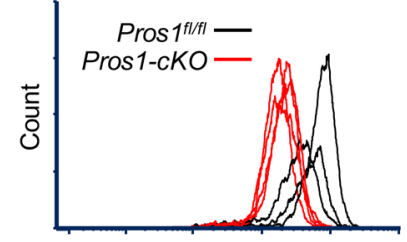

$\zeta$ Chain MFI
C

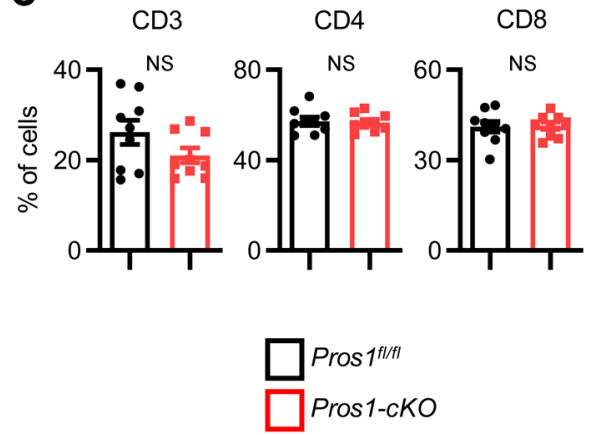

G

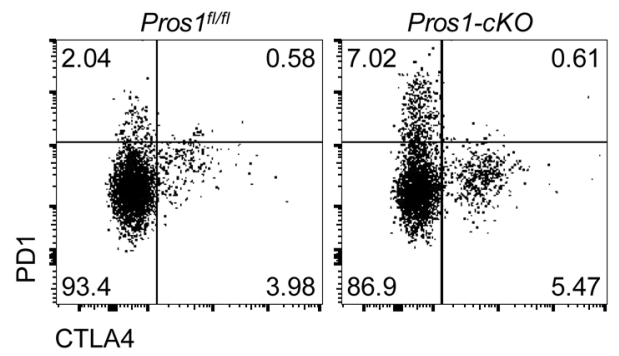

I

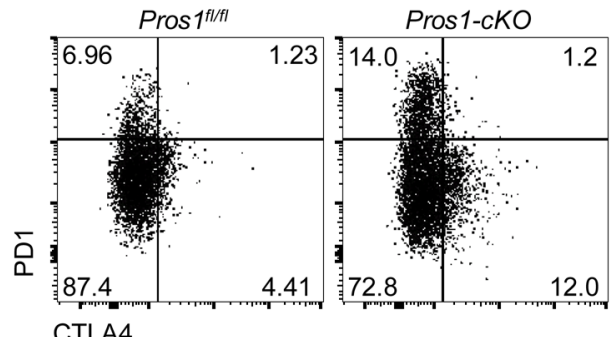

H
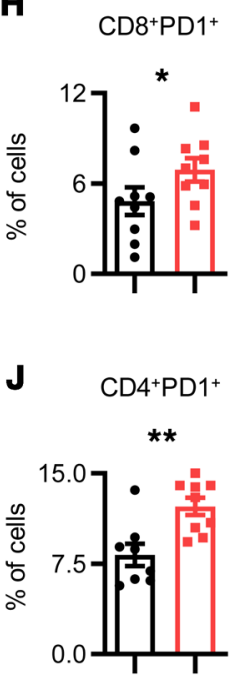

$\mathrm{CD}^{+}{ }^{+} \mathrm{CTLA} 4^{+}$

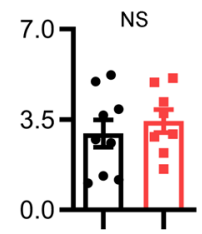

F

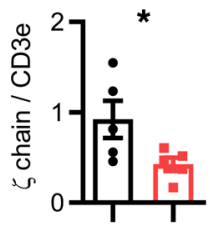

$\mathrm{CD}_{4}{ }^{+} \mathrm{CTLA} 4{ }^{+}$

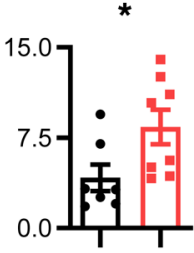

Figure 10. PROS1 inhibition in myeloid cells modulates the T cell response. (A) Ex vivo T cell proliferation assay. Total CD3+ $\mathrm{T}$ cells were left unstimulated (gray bar) or stimulated with anti-CD3/anti-CD28 antibodies and labeled with CellTrace. Stimulated T cells were left alone (green bar) or cocultured with control lgC and F4/80+ macrophages (MФ) sorted from metastatic lungs of control (black bar) or cKO (red bar) mice, purified PROS1 (50 nM, blue bar), or IL-10-neutralizing $\operatorname{IgG}(\alpha \mathrm{lL}-10 ; 5 \mu \mathrm{g} / \mathrm{mL}$, pink bar) for 72 hours. Bars represent the average division index \pm SEM from 2 independent experiments. Each symbol represents a pool of 7-9 mice. (B) Representative FACS plots and quantifications (C) of $C D 3^{+}$or $C D 8^{+}$populations in $C D 45^{+}$gated cells from metastatic lungs of the indicated genotype. Mean percentage \pm SEM; $n=9$ mice/group; 3 independent experiments; $t$ test. (D-J) Impaired activation of metastasis-associated T cells in Pros1-cKO mice. (D) Nitrite levels measured in T cell-macrophage coculture medium, with control lgG or $\alpha$ lL-10 neutralizing antibody. ${ }^{* *} P=0.005$; ${ }^{* * *} P=0.0002 ; 2$-way ANOVA. (E-J) Analysis of T cell receptor (TCR) $\zeta$-chain and the immune checkpoint molecules PD1 and CTLA-4 in T cells from metastatic lungs. (E) Representative FACS histograms show the MFI for $\zeta$-chain from the indicated metastatic lungs analyzed for CD3 $\varepsilon$ and TCR $\zeta$-chain. (F) Relative MFI of $\zeta$-chain/CD3 $\varepsilon$ from 5 mice/group \pm SEM, in 2 independent experiments. ${ }^{*} P=0.026 ; t$ test. (G-J) Representative FACS plots for CD45 ${ }^{+}$CD3 ${ }^{+}$gated cells from the indicated metastatic lungs stained for PD1 and CTLA-4 on $C D 8^{+}(\mathbf{C})$ and $C D 4^{+}(\mathbf{I})$ cell populations and their quantifications ( $\mathbf{H}$ and $\mathbf{J}$, respectively). The mean percentage \pm SEM; $n=8-9$ mice/group; 2 independent experiments. (H) ${ }^{*} P=0.05$ for PD1; (J) ${ }^{*} P=0.019$ for CTLA-4; ${ }^{* *} P=0.0017$ for PD1; $t$ test. NS, nonsignificant.

we identified a metastasis-inhibitory role for PROS1 expressed by BM-derived circulating myeloid cells. Acting as a molecular switch of inflammation, the ablation of PROS1 from this subset of immune cells increases tumor metastatic efficiency in both lung and mammary cancer models. We further revealed that inhibiting PROS1 expression by metastasis-associated macrophages pro- 

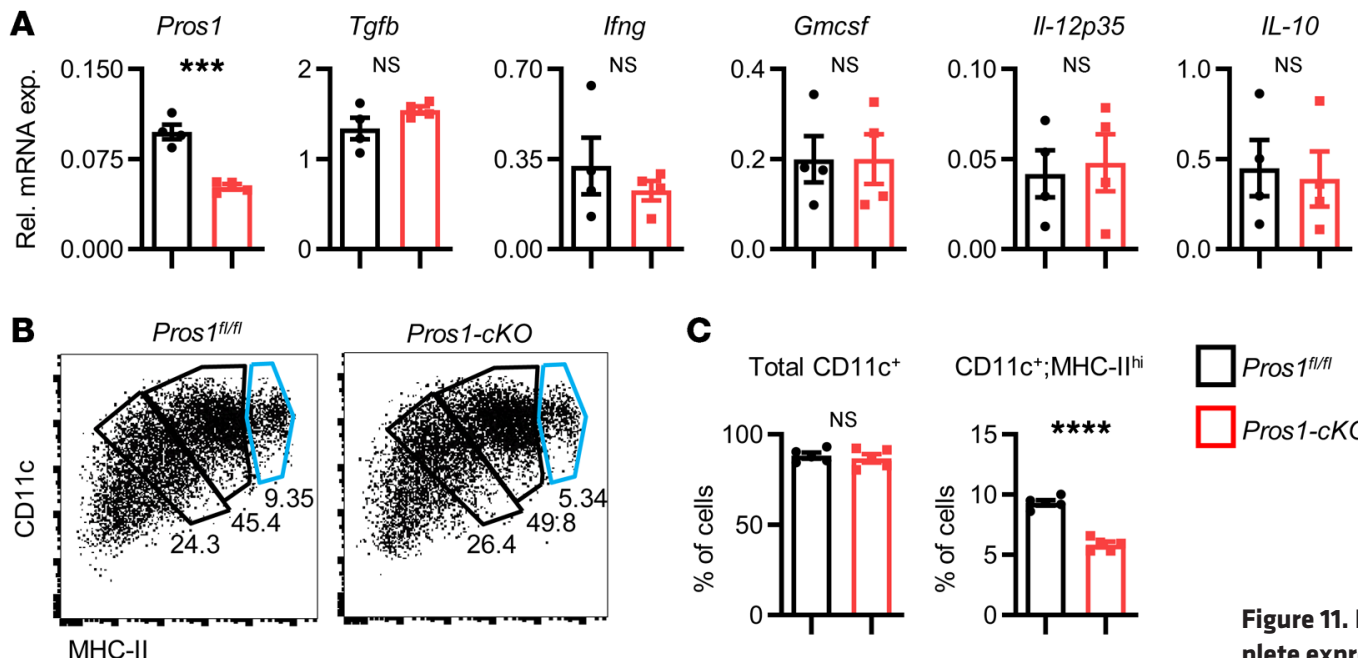

C
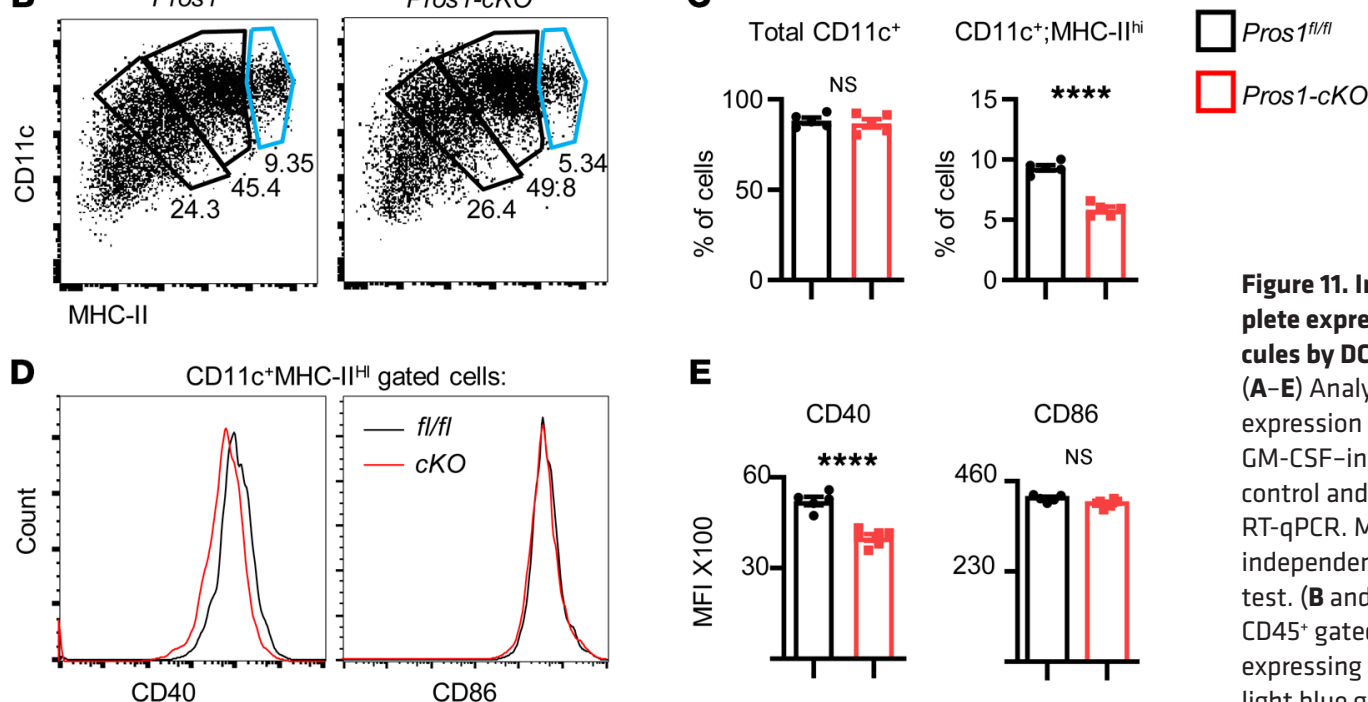

E
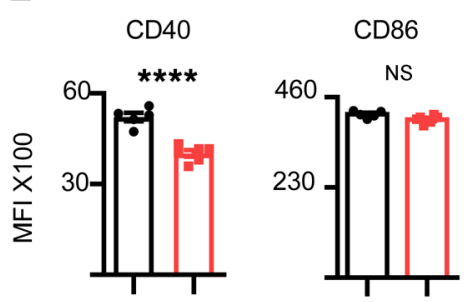

G

CD11 $+M H C-I^{h i}$

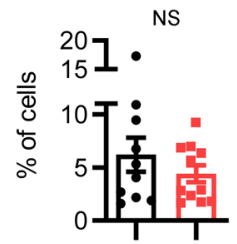

I

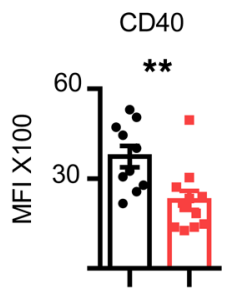

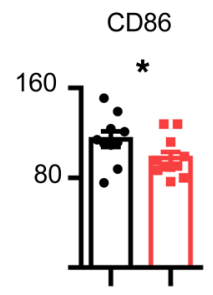

Figure 11. Impaired maturation and incomplete expression of costimulatory molecules by DCs after partial deletion of Pros1. (A-E) Analysis of BMDCs. (A) Relative mRNA expression of the indicated cytokines in GM-CSF-induced BMDCs from Pros $7^{f / f l}$ control and Pros1-cKO mice measured by RT-qPCR. Mean \pm SEM; $n=4$ mice/group; 2 independent experiments. ${ }^{* *} P=0.0002 ; t$ test. (B and $\mathbf{C}$ ) Representative FACS plots of $\mathrm{CD}^{+} 5^{+}$gated cells analyzed for $\mathrm{CD}_{11 c^{+} \text {cells }}$ expressing different MHC-II intensities. The light blue gate indicates the MHC-II ${ }^{\text {hi }}$ cells (B) and quantification (C) of $\mathrm{CD} 45^{+}$gated cells analyzed for total CD11C and CD11C ${ }^{+} \mathrm{MHC}-\mathrm{Ih}^{\mathrm{hi}}$ (light blue gate). Mean percentage \pm SEM; $n=$ 4 mice/group, ${ }^{* * *} P<0.0001$; NS, nonsignificant; $t$ test. (D) Representative histograms and quantification (E) of CD11 ${ }^{+} \mathrm{MHC}-\mathrm{II}^{\text {hi }}$ gated cells (light blue gate) further analyzed for CD40 and CD86 expression. MFI \pm SEM; $n=$ 5-6 mice/group. ${ }^{* * *} P=0.0001 ; t$ test. (F-I) Analysis of DCs from metastatic lungs. (F) Representative FACS plots and (C) quantification of $\mathrm{CD}^{4} 5^{+}$gated cells from control (Pros $7^{\mathrm{fl} /}$ $\left.{ }^{f}\right)$ and cKO metastatic lungs analyzed for total CD11C ${ }^{+} \mathrm{MHC}-$ II $^{\text {hi }}$ cells. Mean percentage \pm SEM; $n=10-11$ mice/group; 2 independent experiments; $t$ test. (H) Representative histograms and (I) quantification of CD11 ${ }^{+} \mathrm{MHC}-\mathrm{II}^{\text {hi }}$ gated cells analyzed for CD40 and CD86 expression. Graphs represent the MFI \pm SEM; $n=10-11$ mice/group. ${ }^{*} P=0.03$ (CD86); ${ }^{*} P=0.003$ (CD40); $t$ test. NS, nonsignificant. motes immune evasion by modulating antitumor responses within T cells, and we highlighted a key role for IL-10 in directly promoting tumor cell invasion and immune suppression.

Our data showed that primary tumor growth was unaffected by PROS1 expressed by circulating myeloid cells (Figure 1, Supplemental Figure 1, and Supplemental Figure 5). Although our in vitro analyses indicate that education with Pros1-cKO BMDMs increased aggressiveness of both LLC and AT3 cells (Figure 6 and Supplemental Figure 6), these cells did not generate larger primary tumors (Supplemental Figure 5), and PROS1 expression in myeloid cells had no effect on their tumor infiltration (Supple- mental Figure 2, A and B). Rather, the education by Pros1-deficient BMDMs was embodied in enhanced metastasis (Figure 5), indicating that different requirements and conditions dictate the impact of myeloid cells on primary tumor versus metastatic loci. In alignment with our results, inhibition of FLT1 kinase activity in host macrophages neither affected macrophage recruitment into metastatic lesions nor to the primary tumor, but instead regulated expression of inflammatory genes in macrophages, promoting metastasis (61). Similarly, TLR2 supports lung metastasis of LLC without affecting primary tumor growth (7). Together with these studies, our data point at distinct behaviors for metastatic and 
primary tumor cells, which may stem from the increased permissiveness and/or synergism provided by the lungs but not at the primary tumor site. Indeed, primary and metastatic sites differ genetically (64) and immunologically $(65,66)$. Given that tumor cells are affected by interactions with immune cells, the differences in immune cell infiltration observed (Figure 2, D-G, and Supplemental Figure 2, A and B) generate distinct immune landscapes in primary and metastatic sites, which in turn may generate environments with differential permissiveness for cancer cell growth. This is supported by elevated MMP9 expression in the lungs of cKO mice, but not of controls (Figure 6, G and $\mathrm{H}$ ).

We found that PROS1 functions as a negative regulator of inflammation in macrophages (Figures 4 and 7) in a MERTK-dependent manner (Figure 9 and Supplemental Figure 7), and that PROS1 neutralization led to enhanced tissue inflammation and consequently increased metastatic seeding (Figure 2 and Supplemental Figure 2). Ablation of PROS1 within BMDMs induced a proinflammatory cytokine signature, mediated through NF- $\kappa \mathrm{B}$ (Figure 4). This pathway is also inhibited by PROS1 in DCs (14) through activation of TAM signaling. Inactivation of TAM signaling in mice led to a hyperactive immune state with an increased inflammatory cytokine signature $(20,67)$. TAMs inhibit inflammation by several mechanisms $(11,13)$, one of which is the phagocytic uptake of apoptotic cells $(11,68)$. Apoptotic cell clearance not only eliminates further inflammation by secondary necrosis of uncleared dying cells $(18,69)$ but also actively stimulates antiinflammatory signaling $(14,15,70,71)$. Within macrophages, MERTK attenuates inflammatory signaling (17), and we recently showed that macrophage-derived PROS1 contributes both to their uptake of apoptotic cells and to dissipating inflammatory cytokine expression in a model of zymosan-induced peritonitis (16).

Along with increased expression of proinflammatory cytokines, PROS1 ablation led to elevated expression of IL-10, a wound healing cytokine that is also upregulated in macrophages after LPS stimulation to regulate the extent of inflammation and resulting tissue damage $(72,73)$. IL-10 is also known for its immunosuppressive capacities $(51,74,75)$ and was recently shown to induce invasion of gastric cancer cells (76). We found that IL-10 secreted by Pros1-deficient BMDMs and metastasis-associated macrophages directly contributed to metastatic progression by inducing LLC invasiveness and survival (Figure 8) and promoting immune suppression by inhibiting $\mathrm{T}$ cell proliferation (Figure 10). In terms of PROS1/TAM signaling within macrophages, lack of TYRO3 expression in BMDMs implies MERTK is the dominant PROS1 receptor (48). Accordingly, MERTK phosphorylation was significantly reduced in Pros1-cKO macrophages (Supplemental Figure 7). Nevertheless, inhibition of MERTK kinase activity reveals a disparity between PROS1-mediated regulation of TNF- $\alpha$ and IL- 6 to that of IL-10 (Figure 9C). This deflection may provide a mechanistic basis for the diverse outcomes observed when different TAM components were inhibited, and is consistent with the inability of TAM kinase inhibitors to block the effect of PROS1 on LLC invasion (Figure 9, A and B), which we found was driven by IL-10 (Figure 8). In vivo, this may be reflected by different TME landscapes dynamically driving distinct molecular pathways. We previously assessed the role of PROS1 in the resolution of inflammation after zymosan A-induced peritonitis (16), where secretion of IL-10 by peritoneal resolution-phase cKO macrophages was lower than in $\mathrm{fl} / \mathrm{fl}$ controls at baseline and after LPS stimulation. Yet, addition of PROS1 suppressed IL-10 production (16). Thus, we propose that the effect of PROS1 on IL-10 expression is context dependent. A potential factor that may modulate the IL-10 response is TNF- $\alpha$, which is necessary for IL-10 expression in inflammatory macrophages (Figure 7, $\mathrm{K}$ and $\mathrm{L}$ ). At the same time, TNF- $\alpha$ is regulated by the PROS1/MERTK axis (Figure 9C), and thus multiple context-dependent factors regulate IL-10 production. Moreover, although PROS1 inhibited LPS-induced IL-10 expression in BMDMs (this study), GAS6 upregulation was reported to enhance IL-10 production under similar conditions (77) in which Pros1 expression was unchanged. Taken together, these results show that regulation of IL-10 by TAM signaling is likely to depend on the functional ligand-receptor interactions in a given physiological context. Further investigations are necessary to elucidate IL-10 regulation by PROS1, including nonkinase functions of MERTK and heterodimers with other receptors (14).

We revealed multiple immunoregulatory roles for PROS1 secreted by metastasis-associated macrophages. In addition to regulating the expression of the immunosuppressive cytokine IL-10 as mentioned above, PROS1 significantly rescued the inhibitory nature of $\mathrm{cKO}$ macrophages (Figure 10A), suggesting that PROS1 stimulates mouse T cells. Indeed, recent studies revealed that PROS1 stimulates human $\mathrm{CD}^{+}(78)$ and $\mathrm{CD}^{+} \mathrm{T}$ cells (79). Moreover, experiments in which IL-10 is neutralized suggest that PROS1 promotes T cell proliferation through IL-10 suppression (Figure 10A). We showed that PROS1 constrained immune suppression in the TME, in that tumor-resident $\mathrm{T}$ cells from $\mathrm{cKO}$ mice had downregulated $\zeta$-chain expression and higher PD1 and CTLA-4 expression (Figure 10). Similarly, inhibiting PROS1 within DCs led to a reduction in maturation and costimulatory molecules (Figure 11), suggesting impaired T cell activation by DCs and further contributing to an immune-suppressive environment. Using a mouse platform, this study is the first to our knowledge to show that in a tumor-related context, PROS1 supports multiple aspects of immune activation, a feature which may be exploited to increase antitumor immune responses.

In addition to their role as negative regulators of inflammation, TAMs are also active proto-oncogenes, where their overexpression stimulates proliferation, migration, cell survival, and resistance to chemotherapy in numerous human cancers (9), pointing to TAMs as attractive targets for anticancer treatment (80-82). As drivers of oncogenesis, TAM ligands GAS6 and PROS1 were shown to stimulate TAM oncogenic signaling within tumor cells $(10,83)$. We previously identified the upregulation of GAS6 by tumor-infiltrating macrophages after education by tumor cells, which in turn fueled tumor growth and metastasis (8). Thus, tumor-infiltrating $\mathrm{BM}$-derived immune cells were educated by tumor cells to upregulate the TAM agonist GAS6, facilitating primary and metastatic growth. Interestingly, PROS1 was not upregulated in these infiltrating tumor macrophages (8). Our present study surprisingly showed that unlike its functional homolog GAS6, PROS1 expression in host macrophages had no effect on LLC and AT3 primary tumor growth (Figure 1 and Supplemental Figures 1 and 5). Unexpectedly, we found that the effect of PROS1 expression by host macrophages on metastasis was opposite to that of GAS6; metastasis was exac- 
erbated by PROS1 ablation, but alleviated by GAS6 deficiency in BM-derived cells. These findings revealed contending roles for the TAM ligands PROS1 and GAS6 within the TME. Varying outcomes were also observed after ablation of different TAMs in the various tumor models tested (11). Reduced tumor growth and metastasis were reported after MERTK inhibition in BM-derived cells in various tumor models, despite their proinflammatory signature (84). This can be explained by the fact that GAS6 upregulation was prevented in MERTK-deficient BM-derived cells or by the observed lack of MERTK-mediated suppression of antitumor immunity (84). Consistent with our observation, loss of both AXL and MERTK in intestine-resident macrophages led to a proinflammatory milieu, favoring a tumor-promoting environment (85). Taken together, our results showed that PROS1 expression in BMDMs potently protected from lung metastasis by inhibiting inflammation.

The effect of BMDM-derived PROS1 on tumor cells did not require direct contact between tumor-infiltrating macrophages and tumor cells. This was demonstrated by efficient metastatic seeding when LLC cells were injected into the tail vein of Pros1-cKO mice, bypassing the primary tumor (Supplemental Figure 2, C-E). It is possible that PROS1-deficient macrophages preferentially migrate to the prospective metastatic sites, where they support and nurture the lungs as a preferred site for future metastasis. This is supported by our BM transplantation experiments, which indicated that $\mathrm{BM}$-derived circulating cells modulated lung inflammation (Figure $3 \mathrm{D}-\mathrm{E}$ ), and suggested that inflammation is a prerequisite for metastatic colonization of the lungs by LLC and AT- 3 cells. Similarly, Qian et al. identified a CCR2- and Gr1-positive distinct population of inflammatory monocytes that are preferentially recruited to metastatic sites over primary tumors, where they promote extravasation of tumor cells into lung parenchyma $(33,34)$.

Regarding PROS1 functions in cancer, this study reveals its functional diversity, which may be context dependent. We previously showed that PROS1 stimulates ERK and AKT phosphorylation in OSCC, contributing to enhanced tumor cell proliferation and migration (10). In contrast with OSCC tumor cells, here we show that the addition of exogenous PROS1 (25 nM) did not stimulate ERK and AKT phosphorylation within LLC and AT-3 cells (Figure 7G). Rather, when added to BMDMs, PROS1 dampened $\mathrm{NF}-\kappa \mathrm{B}-$ mediated inflammatory cytokine production (Figure 7, A-C, and Figure 9C), which in turn attenuated ERK and AKT activation within LLC cells (Figure 7, D-F). Our results point to the PROS1/IL-10 axis in macrophages as a potentially novel pathway where PROS1 directly influences tumor cell survival and invasive potential (Figure 8). Thus, although BMDM-derived PROS1 may potentially stimulate oncogenic pathways in tumor cells via direct TAM activation, it seems the dominant route of tumor cell activation in LLC and AT3 models occurs via inflammation, with IL-10 being a key mediator stimulating ERK, ATK, and STAT3 and facilitating invasion, survival, and metastasis (Figure 8).

Interestingly, tumor-derived PROS1 was recently reported to suppress the immune response in a melanoma model, where its expression affected immune cell infiltration and primary tumor growth (86). Primary tumor growth and its infiltration by immune cells in both lung and mammary models were not affected by PROS1 expression within myeloid cells, suggesting that PROS1-dependent TME responses may vary between tumors. Alternatively, altered biochemical and biological responses may result from different PROS1 variants expressed by tumor and immune cells.

Within BMDMs, PROS1 is a negative regulator of the NF- $\kappa B$ pathway, affecting the secretion of TNF- $\alpha$ and IL-6 (Figure 2, A-C, Figure 4, and Figure 9C). Elevated expression of these cytokines by BMDMs was previously shown to be stimulated by versican, identifying this proteoglycan secreted by LLC cells as a tumor-secreted factor rendering BMDMs prometastatic (7). Our data suggest that loss of PROS1 expression within BMDMs may be sufficient to bypass this versican-dependent prometastatic effect. The supplementation of exogenous PROS1 to BMDM-cKO was sufficient to rescue the metastatic phenotype - reversing metastasis to baseline levels observed in control mice (Figure 7), pointing to BMDM treatment with PROS1 as a putative therapeutic strategy.

In summary, our findings suggest that PROS1 is a key regulator of macrophage inflammatory signaling, also affecting various aspects of immune activation, which together have a strong impact on metastasis. PROS1 expression within macrophages

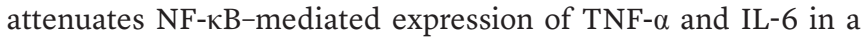
TAM-dependent manner, and induces antitumor immunity both directly and via IL-10, affecting metastatic outcome. IL-10 is revealed as a key cytokine promoting LLC survival and invasiveness as well as immune suppression, and its regulation by PROS1 does not require MERTK kinase activity. The role PROS1 takes in macrophages during inflammation dominates its potential role as a ligand for the proto-oncogenic TAMs, in contrast with what has been previously described for GAS6. Finally, our data provide a strong rationale for utilizing PROS1 to suppress inflammation and stimulate antitumor immunity to restrict metastasis.

\section{Methods}

Detailed experimental methods are provided in Supplemental Methods.

Study approval. All animal studies were approved by the IACUC of the Hebrew University - approval MD-14025-5. Veterinary care was provided to all animals by the Hebrew University animal care facility staff in accordance with AAALAC standard procedures under specific pathogen-free conditions.

\section{Author contributions}

$\mathrm{AM}$ and TBC designed experiments. AM, VLY, KBM, AH, ZT, GM, $\mathrm{M}$ Berger, and SP performed experiments. AM, AH, SMZ, and TBC analyzed data. M Berger, M Baniyash, and SL provided imperative advice in designing experiments and provided critical insight. $\mathrm{M}$ Baniyash provided the anti- $\zeta$-chain antibody. TBC wrote the manuscript. All authors approved the manuscript.

\section{Acknowledgments}

This study was supported by a Research Career Development Award and by a project grant from the Israel Cancer Research Fund (ICRF) to TBC, a research grant by the Israel Cancer Association (ICA) and funded by the Golombo Foundation to TBC, and an ICRF booster grant to AM. SL is funded by a Heisenberg professorship (LO1863/4-1) and by the ubone SPP program (LO1863/5-1) awarded by the German Research Council. Furthermore, SL receives funding from the Margarete-Clemens Stfitung. We thank Nikita Gvardiyan and Shira Kaikov for technical support; and Yaara Tabib, Oded Hayman, Noam Koren, and Avi-Hai Hovav for assisting with 
FACS experiments and analysis. We also thank Hadas Masuri and Zhanna Yekhtin for experimental support and Zvi Granot for providing cancer cell lines. We thank Xiaodong Wang, University of North Carolina, for providing the anti-TAM inhibitors.
Address correspondence to: Tal Burstyn-Cohen, Institute for Dental Sciences, Faculty of Dental Medicine, the Hebrew University, Jerusalem 9112001, Israel. Phone: 972.2.6758582; Email: talbu@mail.huji.ac.il.
1. Hao NB, et al. Macrophages in tumor microenvironments and the progression of tumors. Clin Dev Immunol. 2012;2012:948098.

2. Qian BZ, Pollard JW. Macrophage diversity enhances tumor progression and metastasis. Cell. 2010;141(1):39-51.

3. Quail DF, Joyce JA. Microenvironmental regulation of tumor progression and metastasis. Nat Med. 2013;19(11):1423-1437.

4. Pollard JW. Tumour-educated macrophages promote tumour progression and metastasis. Nat Rev Cancer. 2004;4(1):71-78.

5. Quaranta V, Schmid MC. Macrophage-mediated subversion of anti-tumour immunity. Cells. 2019;8(7):747.

6. Colegio OR, et al. Functional polarization of tumour-associated macrophages by tumourderived lactic acid. Nature. 2014;513(7519):559-563.

7. Kim S, et al. Carcinoma-produced factors activate myeloid cells through TLR2 to stimulate metastasis. Nature. 2009;457(7225):102-106.

8. Loges S, et al. Malignant cells fuel tumor growth by educating infiltrating leukocytes to produce the mitogen Gas6. Blood. 2010;115(11):2264-2273

9. Graham DK, et al. The TAM family: phosphatidylserine-sensing receptor tyrosine kinases gone awry in cancer. Nat Rev Cancer. 2014;14(12):769-785.

10. Abboud-Jarrous G, et al. Protein S drives oral squamous cell carcinoma tumorigenicity through regulation of AXL. Oncotarget. 2017;8(8):13986-14002.

11. Burstyn-Cohen T, Maimon A. TAM receptors, phosphatidylserine, inflammation, and cancer. Cell Commun Signal. 2019;17(1):156-165.

12. Lemke G, Rothlin CV. Immunobiology of the TAM receptors. Nat Rev Immunol. 2008;8(5):327-336.

13. Rothlin CV, et al. TAM receptor signaling in immune homeostasis. Annu Rev Immunol. 2015;33:355-391.

14. Rothlin CV, et al. TAM receptors are pleiotropic inhibitors of the innate immune response. Cell. 2007;131(6):1124-1136.

15. Carrera-Silva EA, et al. T cell-derived protein $S$ engages TAM receptor signaling in dendritic cells to control the magnitude of the immune response. Immunity. 2013;39(1):160-170.

16. Lumbroso D, et al. Macrophage-derived protein $S$ facilitates apoptotic polymorphonuclear cell clearance by resolution phase macrophages and supports their reprogramming. Front Immunol. 2018;9:358.

17. Camenisch TD, et al. A novel receptor tyrosine kinase, Mer, inhibits TNF-alpha production and lipopolysaccharide-induced endotoxic shock. JImmunol. 1999;162(6):3498-503.

18. Cohen PL, et al. Delayed apoptotic cell clearance and lupus-like autoimmunity in mice lacking the c-mer membrane tyrosine kinase. JExp Med. 2002;196(1):135-140.

19. Sen P, et al. Apoptotic cells induce Mer tyrosine kinase-dependent blockade of NF-kappaB activa- tion in dendritic cells. Blood. 2007;109(2):653-660.

20. Lu Q, Lemke G. Homeostatic regulation of the immune system by receptor tyrosine kinases of the Tyro 3 family. Science. 2001;293(5528):306-311.

21. Burstyn-Cohen T, et al. Lack of Protein S in mice causes embryonic lethal coagulopathy and vascular dysgenesis. JClin Invest. 2009;119(10):2942-2953.

22. Clausen BE, et al. Conditional gene targeting in macrophages and granulocytes using LysMcre mice. Transgenic Res. 1999;8(4):265-277.

23. Weiss L, Ward PM. Lymphogenous and hematogenous metastasis of Lewis lung carcinoma in the mouse. Int J Cancer. 1987;40(4):570-574.

24. Pikarsky E, et al. NFאB functions as a tumour promoter in inflammation-associated cancer. Nature. 2004;431(7007):461-466.

25. Ben-Neriah Y, Karin M. Inflammation meets cancer, with NF-kappaB as the matchmaker. Nat Immunol. 2011;12(8):715-723.

26. Karin M. Nuclear factor-kappa B in cancer development and progression. Nature. 2006;441(7092):431-436.

27. Hussell T, Bell TJ. Alveolar macrophages: plasticity in a tissue-specific context. Nat Rev Immunol. 2014;14(2):81-93.

28. Bedoret D, et al. Lung interstitial macrophages alter dendritic cell functions to prevent airway allergy in mice. JClin Invest. 2009;119(12):3723-3738.

29. Tan SYS, Krasnow MA. Developmental origin of lung macrophage diversity. Development. 2016;143(8):1318-1327.

30. Joyce JA, Pollard JW. Microenvironmental regulation of metastasis. Nat Rev Cancer. 2009;9(4):239-252.

31. Gorelik E, et al. Augmentation of metastasis formation by thioglycollate-elicited macrophages. Int J Cancer. 1982;29(5):575-581.

32. Nowicki A, et al. Impaired tumor growth in colony-stimulating factor 1 (CSF-1)-deficient, macrophage-deficient op/op mouse: evidence for a role of CSF-1-dependent macrophages in formation of tumor stroma. Int JCancer. 1996;65(1):112-119.

33. Qian BZ, et al. CCL2 recruits inflammatory monocytes to facilitate breast-tumour metastasis. Nature. 2011;475(7355):222-225.

34. Qian B, et al. A distinct macrophage population mediates metastatic breast cancer cell extravasation, establishment and growth. PLoS One. 2009;4(8):e6562.

35. Lin EY, et al. Colony-stimulating factor 1 promotes progression of mammary tumors to malignancy. J Exp Med. 2001;193(6):727-739.

36. Shand FHW, et al. Tracking of intertissue migration reveals the origins of tumor-infiltrating monocytes. Proc Natl Acad Sci USA. 2014;111(21):7771-7776.

37. Sharma SK, et al. Pulmonary alveolar macrophages contribute to the premetastatic niche by suppressing antitumor $\mathrm{T}$ cell responses in the lungs. JImmunol. 2015;194(11):5529-5538.

38. Kratofil RM, et al. Monocyte conversion during inflammation and injury. Arterioscler Thromb Vasc Biol. 2017;37(1):35-42.

39. Matute-Bello G, et al. Optimal timing to repopulation of resident alveolar macrophages with donor cells following total body irradiation and bone marrow transplantation in mice. J Immunol Methods. 2004;292(1-2):25-34.

40. Deng T, et al. Toll-like receptor-mediated inhibition of Gas 6 and ProS expression facilitates inflammatory cytokine production in mouse macrophages. Immunology. 2012;135(1):40-50.

41. van der Meer JHM, et al. TAM receptors, Gas6, and protein S: roles in inflammation and hemostasis. Blood. 2014;123(16):2460-2469.

42. Burstyn-Cohen T, et al. Genetic dissection of TAM receptor-ligand interaction in retinal pigment epithelial cell phagocytosis. Neuron. 2012;76(6):1123-1132.

43. Mantovani A, et al. Cancer-related inflammation. Nature. 2008;454(7203):436-444.

44. Coussens LM, Werb Z. Inflammation and cancer. Nature. 2002;420(6917):860-867.

45. Merchant N, et al. Matrix metalloproteinases: their functional role in lung cancer. Carcinogenesis. 2017;38(8):766-780.

46. Chou CH, et al. MMP-9 from sublethally irradiated tumor promotes Lewis lung carcinoma cell invasiveness and pulmonary metastasis. Oncogene. 2012;31(4):458-468.

47. Zeni E, et al. Macrophage expression of interleukin-10 is a prognostic factor in nonsmall cell lung cancer. Eur Respir J. 2007;30(4):627-632.

48. Zagórska A, et al. Diversification of TAM receptor tyrosine kinase function. Nat Immunol. 2014;15(10):920-928.

49. Lee Y-J, et al. Inhibiting Mer receptor tyrosine kinase suppresses STAT1, SOCS1/3, and NF- $\kappa \mathrm{B}$ activation and enhances inflammatory responses in lipopolysaccharide-induced acute lung injury. J Leukoc Biol. 2012;91(6):921-932.

50. Noy R, Pollard JW. Tumor-associated macrophages: from mechanisms to therapy. Immunity. 2014;41(1):49-61.

51. Vukmanovic S, et al. T-cell activation is enhanced by targeting IL-10 cytokine production in toll-like receptor-stimulated macrophages. Immunotargets Ther. 2012;1:13-23.

52. Sharma S, et al. T cell-derived IL-10 promotes lung cancer growth by suppressing both T cell and APC function. JImmunol.1999;163(9):5020-5028.

53. Jacobs F, et al. IL-10 up-regulates nitric oxide (NO) synthesis by lipopolysaccharide (LPS)-activated macrophages: improved control of Trypanosoma cruzi infection. Clin Exp Immunol. 1998;113(1):59-64.

54 . Sato $\mathrm{K}$, et al. Nitric oxide plays a critical role in suppression of T-cell proliferation by mesenchymal stem cells. Blood. 2007;109(1):228-234.

55. Ridnour LA, et al. NOS inhibition modulates immune polarization and improves radiation-induced tumor growth delay. Cancer Res. 
2015;75(14):2788-2799.

56. Baniyash M. TCR $\zeta$-chain downregulation: curtailing an excessive inflammatory immune response. Nat Rev Immunol. 2004;4(9):675-687.

57. Preynat-Seauve O, et al. Tumor-infiltrating dendritic cells are potent antigen-presenting cells able to activate T cells and mediate tumor rejection. JImmunol. 2006;176(1):61-67.

58. Kaplan RN, et al. Preparing the "soil": the premetastatic niche. Cancer Res. 2006;66(23):11089-11093.

59. Hanna RN, et al. Patrolling monocytes control tumor metastasis to the lung. Science. 2015;350(6263):985-990.

60. Kaplan RN, et al. VEGFR1-positive haematopoietic bone marrow progenitors initiate the pre-metastatic niche. Nature. 2005;438(7069):820-827.

61. Qian BZ, et al. FLT1 signaling in metastasis-associated macrophages activates an inflammatory signature that promotes breast cancer metastasis. J Exp Med. 2015;212(9):1433-1448.

62. Hiratsuka S, et al. MMP9 induction by vascular endothelial growth factor receptor- 1 is involved in lung-specific metastasis. Cancer Cell. 2002;2(4):289-300.

63. Yang M, et al. Tumor cell-activated CARD9 signaling contributes to metastasis-associated macrophage polarization. Cell Death Differ. 2014;21(8):1290-1302.

64 . Wu J, et al. Genetic differences between primary and metastatic tumors from cross-institutional data. JClin Oncol. 2018;36(15 suppl):e18572.

65. Szekely B, et al. Immunological differences between primary and metastatic breast cancer. Ann Oncol. 2018;29(11):2232-2239.

66. Kim R, et al. Differences in tumor microenviron- ments between primary lung tumors and brain metastases in lung cancer patients: therapeutic implications for immune checkpoint inhibitors. BMC Cancer. 2019;19(1):19.

67. Lemke G, Lu Q. Macrophage regulation by Tyro 3 family receptors. Curr Opin Immunol. 2003;15(1):31-36.

68. Lemke G, Burstyn-Cohen T. TAM receptors and the clearance of apoptotic cells. Ann N Y Acad Sci. 2010;1209(1):23-29.

69. Elliott MR, Ravichandran KS. Clearance of apoptotic cells: implications in health and disease. JCell Biol. 2010;189(7):1059-1070.

70. Lee CS, et al. Boosting apoptotic cell clearance by colonic epithelial cells attenuates inflammation in vivo. Immunity. 2016;44(4):807-820.

71. Fadok VA, et al. Macrophages that have ingested apoptotic cells in vitro inhibit proinflammatory cytokine production through autocrine/paracrine mechanisms involving TGF- $\beta$, PGE2, and PAF. $J$ Clin Invest. 1998;101(4):890-898.

72. Pengal RA, et al. Lipopolysaccharide-induced production of interleukin-10 is promoted by the serine/threonine kinase Akt. Mol Immunol. 2006;43(10):1557-1564.

73. Ernst O, et al. Exclusive temporal stimulation of IL-10 expression in LPS-stimulated mouse macrophages by cAMP inducers and type I interferons. Front Immunol. 2019;10(1788):1788.

74. Mittal SK, Roche PA. Suppression of antigen presentation by IL-10. Curr Opin Immunol. 2015;34:22-27.

75. Jung K, et al. Ly6Clo monocytes drive immunosuppression and confer resistance to anti-VEGFR2 cancer therapy. JClin Invest. 2017;127(8):3039-3051.
76. Chen L, et al. IL-10 secreted by cancer-associated macrophages regulates proliferation and invasion in gastric cancer cells via c-Met/STAT3 signaling. Oncol Rep. 2019;42(2):595-604.

77. Kim SY, et al. Liver X receptor and STAT1 cooperate downstream of Gas6/Mer to induce anti-inflammatory arginase 2 expression in macrophages. Sci Rep. 2016;6(1):1-16.

78. Cabezón R, et al. MERTK as negative regulator of human T cell activation. J Leukoc Biol. 2015;97(4):751-760.

79. Peeters MJW, et al. MERTK Acts as a costimulatory receptor on human $\mathrm{CD}^{+} \mathrm{T}$ cells. Cancer Immunol Res. 2019;7(9):1472-1484.

80. Sheridan C. First Axl inhibitor enters clinical trials. Nat Biotechnol. 2013;31(9):775-776.

81. Verma A, et al. Targeting Axl and Mer kinases in cancer. Mol Cancer Ther. 2011;10(10):1763-1773.

82. Schlegel J, et al. MERTK receptor tyrosine kinase is a therapeutic target in melanoma. J Clin Invest. 2013;123(5):2257-2267.

83. Shiozawa Y, et al. GAS6/AXL axis regulates prostate cancer invasion, proliferation, and survival in the bone marrow niche. Neoplasia. 2010;12(2):116-127.

84. Cook RS, et al. MerTK inhibition in tumor leukocytes decreases tumor growth and metastasis. J Clin Invest. 2013;123(8):3231-3242.

85. Bosurgi L, et al. Paradoxical role of the proto-oncogene Axl and Mer receptor tyrosine kinases in colon cancer. Proc Natl Acad Sci. 2013;110(32):13091-13096.

86. Ubil E, et al. Tumor-secreted Pros1 inhibits macrophage M1 polarization to reduce antitumor immune response. J Clin Invest. 2018;128(6):2356-2369. 